\title{
From Shortest-path to All-path: The Routing Continuum Theory and its applications
}

\author{
Yanhua Li, Member, IEEE, Zhi-Li Zhang, Fellow, IEEE, and Daniel Boley, Member, IEEE
}

\begin{abstract}
As a crucial operation, routing plays an important role in various communication networks. In the context of data and sensor networks, routing strategies such as shortest-path, multi-path and potential-based ("all-path") routing have been developed. Existing results in the literature show that the shortest path and all-path routing can be obtained from $L_{1}$ and $L_{2}$ flow optimization, respectively. Based on this connection between routing and flow optimization in a network, in this paper we develop a unifying theoretical framework by considering flow optimization with mixed (weighted) $L_{1} / L_{2}$-norms. We obtain a surprising result: as we vary the trade-off parameter $\theta$, the routing graphs induced by the optimal flow solutions span from shortest-path to multi-path to all-path routing - this entire sequence of routing graphs is referred to as the routing continuum. We also develop an efficient iterative algorithm for computing the entire routing continuum. Several generalizations are also considered, with applications to traffic engineering, wireless sensor networks, and network robustness analysis.
\end{abstract}

Index Terms—Routing continuum, network flow, betweenness centrality.

\section{INTRODUCTION}

Routing is a crucial operation in many types of networks from communication networks to transportation networks. For instance, in modern IP-based data networks, shortest path routing is most commonly used. In traditional telecommunication networks, dynamic alternative routing strategies that employ paths that are longer than shortest paths have been also proposed to reduce call blocking probabilities (see, e.g., [1], [17]). In wireless networks, due to the unstable channel characteristics, using a single "shortest" path (e.g., with link quality as link weights) for routing is often not the best choice; routing strategies that go beyond shortest path routing (see, e.g., [4], [16], [22], [31] and references therein) using multiple paths are often more effective. In the other extreme, in wireless sensor networks - due to their power and other resource constraints - potential-based routing [26] has been proposed, where the source essentially utilizes all (eligible) paths to transmit data to the destination. In [26], it is shown that such "all-path" routing minimizes the total energy dissipation of routing and thus maximizes the network lifetime. Clearly, what routing strategies to employ in a network hinges on what objectives are important in practice, therefore should be optimized. However, from a theoretical perspective, when using multi-path routing that goes beyond a single shortest path, two questions arise: i) what set of paths should be used for routing? and ii) how traffic should be split (and merged) at any node along the multiple paths, especially when the paths are not all disjoint?

In addressing these questions, in this paper we consider routing as flow optimization in a network. Our idea is inspired by the earlier results where it has been shown that shortest path routing can be derived from network flow optimization

- This work was supported in part by the NSF grants CNS-1017092 and IIS-0916750, the DTRA grant HDTRA1-09-1-0050. An earlier version of this work appeared in the Proceedings of IEEE ICDCS [24], June 2011. Yanhua Li is with HUAWEI Noah's Ark Lab, China. Zhi-Li Zhang and Daniel Boley are with the Department of Computer Science and Engineering, University of Minnesota, Twin Cities, Minneapolis, MN, 55455.

E-mail: \{yanhua,zhzhang,boley\}@cs.umn.edu with $L_{1}$ norm [35], whereas potential-based, "all-path" routing can be derived from network flow optimization with $L_{2}$-norm objective [17], [26]. We introduce the network flow optimization problem, with mixed $L_{1} / L_{2}$-norm objective, which intuitively can be interpreted as a trade-off between the latency and energy dissipation of paths used for routing (collectively, the paths form a routing graph): shorter paths lead to better routing with low latency, while diffusing traffic along more paths generally reduces energy dissipation. Using this formulation, we obtain a surprising result: as we vary the tradeoff parameter $\theta$, the routing graphs induced by the optimal flow solutions span from the shortest-path routing to multipath routing with increasing path lengths to the potentialbased ("all-path") routing - this entire (finite) sequence of routing graphs is referred to as the routing continuum. Our theory therefore subsumes the earlier $L_{1}$ and $L_{2}$ network flow optimization results [17], [35] as two extreme points in the entire routing continuum.

Furthermore, by considering the dual of the mixed $L_{1} / L_{2}$ norm network flow optimization problem, we develop an efficient iterative algebraic process as well as algorithms for identifying precisely the boundary conditions separating the finite sequence of routing graphs, and for computing the entire routing continuum and optimal flow solutions $X^{*}(\theta)$ for any $\theta \geq 0$. In particular, $X^{*}(\theta)$ specifies how traffic should be split and merged in the induced routing graph. We also generalize the theory to account for multiple flows (traffic demands), link capacity constraints and heterogeneous $L_{1} / L_{2}$ link weights, with applications to traffic engineering and wireless sensor networks. For instance, given a set of link weights and traffic demands on a network, our theory can be used to find the "best" routing graph (i.e., the best mix of shorter and longer paths) that minimizes the overall maximum link utilization.

In summary, our contributions are i) we develop a unifying theory using mixed $L_{1} / L_{2}$-norm network flow optimization and show that it can generate the entire routing continuum from shortest-path to "all-path" routing; ii) we develop an efficient iterative process for computing the entire routing 
continuum and optimal flow solutions $X^{*}(\theta)$ for any $\theta \geq 0$; iii) the basic theory is further generalized to account for multiple flows (traffic demands), link capacity constraints and heterogeneous $L_{1} / L_{2}$ link weights, with applications to traffic engineering and wireless sensor networks. iv) Moreover, by applying the routing continuum theory, we generalize the betweenness centrality measure using mixed network flow, with applications in network robustness analysis. Last but not the least, while we focus on network routing in this paper, we believe that our results can be applied to many other applications where the problems can be cast in terms of flows in a network. Going beyong a preliminary version of this work [24], we provide numerical analysis using a real network topology, i.e., the Abilene network topology, to illustrate the routing continuum theory. Moreover, we discuss an application of the routing continuum theory in analyzing network robustness. Lastly, in section 3.1, we introduce new theoretical results that the mixed $L_{1}$ - and $L_{2}$-norm network flow problem can achieve the optimal trade-off between the average delay and the average energy consumption in the network. Due to the limited space, we delegate parts of these results to the supplementary file [25] of the paper.

The remainder of the paper is organized as follows. In Section 2 we introduce the basic notations and re-state the known $L_{1}$ and $L_{2}$ flow optimizations using our notations. In Section 3 the general theory and results using the mixed $L_{1} / L_{2}$-norm flow optimization are established, and the iterative computation process and algorithms are described in Section 4. In Section 5, we consider several generalizations, with applications to traffic engineering, wireless sensor networks, and network robustness analysis. Section 6 discusses the related work, and the paper is concluded in Section 7.

\section{Shortest Path and "All-Path" Routing as Network Flow Optimization}

In this section, we first introduce the basic notations that will be used throughout the paper. Then, we illustrate how shortest path routing and potential-based "all-path" routing can be formulated as the flow optimization problems in a network using metric norms (on the flow space). More specifically, the shortest path routing results from minimizing the (weighted) $L_{1}$-norm of flows between a given source-destination pair in a network, whereas the potential-based, "all-path" routing results from minimizing the corresponding $L_{2}$-norm.

\subsection{Network and Flows: Basic Notations}

We represent a $n$-node network as an undirected, weighted graph, $G=(V, E, W)$, where $V=\{1,2, \ldots, n\}$ is the set of vertices, $E$ is the set of edges, and each edge $(i, j) \in E$ is assigned a positive weight $w_{i j}$. $W$ is an $n \times n$ matrix, where each $(i, j)$-th entry denotes the link weight $w_{i j}$. As $G$ is undirected, $(i, j)$ and $(j, i)$ represent the same edge in $E$, and $w_{i j}=w_{j i}>0$. Define $w_{i j}=0$ if $(i, j) \notin E$, then the weight matrix $W=\left[w_{i j}\right]$ is symmetric. In particular, if all edges have a unit weight, i.e., $W$ is a $0-1$ matrix, then $G$ represents a simple graph, and $W$ is the corresponding adjacency matrix.

Let $d=[s, t], s, t \in V, s \neq t$, denote a source-destination (or source-sink) pair in the network $G$. A flow of $I^{(d)}$ unit amount that flows from source $s$ to destination $t$ is mathematically defined as a function, $X^{(d)}: V \times V \rightarrow \mathbb{R}^{+}$ $\left(\mathbb{R}^{+}\right.$is the set of non-negative real numbers), satisfying the following constraints:

$$
\text { along one direction: } \quad \text { if } X_{i j}^{(d)}>0 \text { then } X_{j i}^{(d)}=0,
$$

along network edges:

$$
\text { if }(i, j) \notin E \text { then } X_{i j}^{(d)}=0 \text {, }
$$

flow conservation at $s: \quad I^{(d)}+\sum_{k=1}^{n} X_{k s}^{(d)}=\sum_{j=1}^{n} X_{s j}^{(d)},(3)$

intermediate node $i \neq s, t: \quad \sum_{k=1}^{n} X_{k i}^{(d)}=\sum_{j=1}^{n} X_{i j}^{(d)}$,

at destination $t: \quad \sum_{k=1}^{n} X_{k t}^{(d)}=\sum_{j=1}^{n} X_{t j}^{(d)}+I^{(d)}$.(5)

Note that in this flow definition, for each (undirected) edge $(i, j) \in E$, both $X_{i j}^{(d)}$ and $X_{j i}^{(d)}$ are defined, and the constraint in eq.(1) states that if $X_{i j}^{(d)}>0$, then $X_{j i}^{(d)}=0$; or if $X_{j i}^{(d)}>$ 0 , then $X_{i j}^{(d)}=0$. It is possible that for $(i, j) \in E$, both $X_{i j}^{(d)}=X_{j i}^{(d)}=0$. In particular, by the constraint in eq.(2), $X_{i j}^{(d)}=X_{j i}^{(d)}=0$ for $(i, j) \notin E$. The flow constraints in eqs.(3)-(5) state that an amount of $I^{(d)}$ units of flow is injected at source $s$, and the same amount is removed from destination $t$, while the amount of flow entering any intermediate node $i$ is the same as the amount leaving the node.

Given a flow $X^{(d)}$ between a source-destination pair $d=$ $[s, t]$, it induces an oriented (or directed) sub-graph of $G$, $G_{X^{(d)}}=\left(V_{X^{(d)}}, E_{X^{(d)}}\right)$, where an $\operatorname{arc}\langle i, j\rangle \in E_{X^{(d)}}$ and $i, j \in V_{X^{(d)}}$ if and only if $X_{i j}^{(d)}>0$. As a directed acyclic graph (DAG) between $s$ and $t, G_{X^{(d)}}$ represents the routes used to route the flow $X^{(d)}$ (of $I^{(d)}$ units) from source $s$ to destination $t$, and we refer to it as the routing graph for the flow $X^{(d)}$. When $G_{X^{(d)}}$ consists of more than a single path between $s$ and $t$, then $X_{i j}^{(d)}$ indicates how much flow is routed along the edge (arc) $\langle i, j\rangle$. In general, the flow may be split or merged $^{1}$ at nodes in $G_{X^{(d)}}$, and routed along different paths between $s$ and $d$. We will use $\mathcal{F}^{(d)}$ to denote the collection of flows, i.e., all functions that satisfy eqs.(2)-(5).

In the next two subsections we will use two well-known results [17], [35] to illustrate that certain common routing strategies, namely, shortest path routing and potential-based, "allpath" routing, can be derived by minimizing the (weighted) $L_{1}$-norm and $L_{2}$-norm, respectively, of flows between a given source-destination pair in a network. In Section 3 we will generalize these results and establish that by minimizing flows using mixed $L_{1}$-norm and $L_{2}$-norm, we can generate a continuиm of routing strategies, resulting in a sequence of routing graphs with varying numbers of paths of differing costs selected, from the shortest paths to all paths (between a sourcedestination pair). Table 1 provides notations used in the paper.

\subsection{Shortest-Path Routing \& $L_{1}$-norm Flow Optimization}

Without loss of generality, unless otherwise specified, we assume that $s=1$ and $t=n$, and $I^{(d)}=1$. For clarity of notation, we drop the superscript $d$ from $X^{(d)}$. In other

1. The flow definition implicitly assumes that flows are "infinitely divisible fluid" - they can be split and merged arbitrarily at any node of the network, as long as the above flow conservation constraints are met. This mathematical definition of network flows thus provides an idealized (fluid) abstraction of, e.g., traffic demands routed from a source to a destination in a communication network, or commodities transported from a source to a destination in a transportation network, and so forth. 
TABLE 1

Notations

\begin{tabular}{|c|c|}
\hline$G=(V, E, W)$ & $\begin{array}{l}G \text { is an undirected graph, with link weight } \\
\text { matrix } W \text {, with }|V|=n \text {. }\end{array}$ \\
\hline$d=[s, t]$ & $\begin{array}{l}d \text { is a network demand, with source } s \text { and } \\
\text { destination } t \text {. }\end{array}$ \\
\hline$\theta$ & $\begin{array}{l}\text { Trade-off parameter between } L_{1} \text { - and } L_{2}- \\
\text { norm objectives in the mixed network flow } \\
\text { optimization problem eq.(18). }\end{array}$ \\
\hline $\begin{array}{l}X^{(d)}=\left[X_{i j}^{(d)}\right] \\
X=\left[X_{i j}\right] \\
X^{*}(\theta)=\left[X_{i j}^{*}(\theta)\right]\end{array}$ & $\begin{array}{l}X^{(d)} \text { is the network flow matrix for the } \\
\text { demand } d \text {, where each entry } X_{i j}^{(d)} \text { rep- } \\
\text { resents the flow distributed on the edge } \\
\langle i, j\rangle \text {. When } d=[1, n] \text { is considered, the } \\
\text { superscript }(d) \text { is omitted. } X^{*}(\theta) \text { is the } \\
\text { optimal flow for the mixed network flow } \\
\text { optimization problem eq. (18). }\end{array}$ \\
\hline $\begin{array}{l}U=\left[U_{i}\right] \\
U^{*}(\theta)=\left[U_{i}^{*}(\theta)\right]\end{array}$ & $\begin{array}{l}U_{i} \text { 's are the Lagrange multipliers of } \\
\text { the flow conservation constraints eq. }(7) \text {. } \\
U_{i}^{*}(\theta) \text { 's are the optimal values of } U_{i} \text { 's } \\
\text { to the mixed flow optimization problem } \\
\text { eq.(18). }\end{array}$ \\
\hline $\begin{array}{l}\mathcal{R}(\theta), \mathcal{R}(0) \\
\mathcal{R}(\infty)\end{array}$ & $\begin{array}{l}\mathcal{R}(\theta) \text { is the routing graph induced by } \\
X^{*}(\theta) \text {. In particular, } \mathcal{R}(0) \text { and } \mathcal{R}(\infty) \text { are } \\
\text { the routing graphs for "all-path" routing } \\
\text { and shortest path routing, respectively. }\end{array}$ \\
\hline $\begin{array}{l}\Delta^{(+)}(\theta)=\left[\Delta_{i}^{(+)}(\theta)\right] \\
\Delta^{(-)}(\theta)=\left[\Delta_{i}^{(-)}(\theta)\right] \\
\Delta(\theta)=\left[\Delta_{i}(\theta)\right]\end{array}$ & $\begin{array}{l}\Delta_{i}^{(+)}(\theta) \text { (resp. } \Delta_{i}^{(-)}(\theta) \text { ) is the number of } \\
\text { incoming (resp. outgoing) edges of node } \\
i \text { with no-zero flow in } X^{*}(\theta) . \Delta_{i}(\theta)= \\
\Delta_{i}^{(-)}(\theta)-\Delta_{i}^{(+)}(\theta) .\end{array}$ \\
\hline
\end{tabular}

words, the flow $X$ (as a function) is equivalently specified by a set of $n^{2}$ variables, $X_{i j}$ 's, $1 \leq i, j \leq n$.

Consider the following $L_{1}$-norm network flow optimization problem, which can be solved using linear programming (LP). $L_{1}$-norm Network Flow Optimization ( $L_{1}$ Primal):

$$
\min _{X} \sum_{i=1}^{n} \sum_{j=1}^{n} w_{i j} X_{i j}
$$

subject to the flow conservation constraints eqs.(2)-(5), which are more compactly represented below using $X_{i j}$ 's:

$$
\sum_{j:(i, j) \in E} X_{i j}-\sum_{k:(k, i) \in E} X_{k i}= \begin{cases}1 & \text { if } i=1 \\ 0 & \text { if } i=2, \ldots, n-1 \\ -1 & \text { if } i=n\end{cases}
$$

and $X_{i j} \geq 0,1 \leq i, j \leq n$.

Note that the feasible solutions to eq.(6) subject to eqs.(7) and (8) satisfy constraints eqs.(2)-(5), and an optimal solution to this must also satisfy eq.(1) automatically. Hence without loss of generality, when considering the optimization in eq.(6), we can restrict ourselves to $X$ 's that are flows, i.e., $X \in \mathcal{F}$. Thus we can re-state the optimization in eq.(6) as

$$
\min _{X \in \mathcal{F}} \sum_{i}^{n} \sum_{j}^{n} w_{i j} X_{i j}
$$

In other words, the optimization solution to eq.(6) is the flow that minimizes the weighted $L_{1}$-norm.

To show that the optimal solution to this $L_{1}$-norm network flow optimization gives rise to the shortest-path routing, we consider its dual, stated below in terms of the Lagrange multipliers $-U_{i}$ 's (corresponding to the flow conservation constraints eq. $\left.(7)^{2}\right)$ :

Dual of $L_{1}$-norm Network Flow Optimization ( $L_{1}$ Dual):

$$
\begin{gathered}
\max _{U} U_{1} \\
\text { subject to } U_{n}=0 \text { and } U_{i}-U_{j} \leq w_{i j}, \forall(i, j) \in E .
\end{gathered}
$$

Let $X^{*}$ denote the optimal flow solution to the primal problem eq.(6), and $U^{*}$ the optimal solution to the dual problem. The duality and complementary slackness give us the following relations between $X_{i j}^{*}$ 's and $U_{i}^{*}$ 's ( $c f$. Lemma 1 in [35] and the transportation and network flow problems in Chapter 5 in [27]).

$$
\begin{aligned}
\text { if } X_{i j}^{*} & >0 \text {,then } U_{i}^{*}-U_{j}^{*}=w_{i j} ; \\
\text { and if } X_{i j}^{*} & =0 \text {, then } U_{i}^{*}-U_{j}^{*}<w_{i j} .
\end{aligned}
$$

Using these relations, the authors in [35], show that the optimal solution to the dual problem, $U_{i}^{*}$ 's, have the following properties ( $c f$. Theorem 1 and its proof in [35]):

LEMMA 1. Let $P$ be a path from node 1 to node $n$. If for each edge (arc) $\langle i, j\rangle \in P, U_{i}^{*}-U_{j}^{*}=w_{i j}$, then $P$ is a shortest path from node 1 to node $n$ (with respect to the weights $w_{i j}$ 's), and $U_{1}^{*}=\sum_{\langle i, j\rangle \in P} w_{i j}$. Alternatively, if $Q$ is a path from node 1 to node $n$ that is not a shortest path, then $U_{1}^{*}<$ $\sum_{\langle i, j\rangle \in Q} w_{i j}$.

The above lemma implies that for any node $i$ on a shortest path, $U_{i}^{*}$ is the shortest-path distance from node $i$ to node $n$ (the destination). Furthermore, the optimal flow $X^{*}$ is only routed along the shortest paths between source 1 and destination $n$. In other words, the resulting routing graph $G_{X}$ * is the DAG formed by the shortest paths from 1 to $n$ only. When there are multiple shortest paths between 1 and $n, X_{i j}^{*}$ specifies the amount of flow carried on the edges of node $i$ that are on the shortest paths, thus how the flow should be split among multiple shortest path at node $i$.

\subsection{Potential-based (“All-path”) Routing and $L_{2}$-norm Flow Optimization}

We now consider the following (weighted) $L_{2}$-norm network flow optimization problem:

$L_{2}$-norm Network Flow Optimization ( $L_{2}$ Primal):

$$
\min _{X \in \mathcal{F}} \sum_{i=1}^{n} \sum_{j=1}^{n} w_{i j} X_{i j}^{2} \text {. }
$$

To show that the optimal solution to this $L_{2}$-norm network flow optimization gives rise to the potential-based, "all-path" routing, we again consider its dual, stated below in terms of the Lagrange multipliers $U_{i}$ 's (where for convenience we have used $-2 U_{i}$ 's as the multipliers for the flow conservation constraints eq.(7)), where the proof is similar to that in [17], and we omit it here:

Dual of $L_{2}$-norm Network Flow Optimization ( $L_{2}$ Dual):

$$
\max _{U} U_{1}-\frac{1}{2} \sum_{i=1}^{n} \sum_{j: U_{i}>U_{j}} \frac{\left(U_{i}-U_{j}\right)^{2}}{w_{i j}} .
$$

2. Note that our Lagrange multipliers are negatives of those used in the "Dual Shortest Path Formulation (D-SP)" in [35], p. 3. 
subject to $U_{n}=0$.

Let $X^{*}$ denote the optimal flow solution to the primal problem eq.(13), and $U^{*}$ the optimal solution to the dual problem. The duality and complementary slackness give us the following relations between $X_{i j}^{*}$ 's and $U_{i}^{*}$ 's: for any edge $(i, j) \in E$,

$$
\begin{aligned}
& \text { if } U_{i}^{*}>U_{j}^{*} \text {, then } X_{i j}^{*}=\frac{U_{i}^{*}-U_{j}^{*}}{w_{i j}}>0 ; \\
& \text { and if } U_{i}^{*} \leq U_{j}^{*} \text {, then } X_{i j}^{*}=0 .
\end{aligned}
$$

If we treat $w_{i j}$ as the resistance on edge $(i, j) \in E$, then the relation eq.(15) gives us precisely Ohm's law [17], and $U_{i}^{*}$ is the voltage (potential) at node $i$ when a unit of current is injected at source node 1 and removed at sink node $n$ (and grounded with $\left.U_{n}^{*}=0\right)$. For any $(i, j) \in E$, if $U_{i}^{*}>U_{j}^{*}$, then the current $I_{i j}$ flowing from node $i$ to node $j$ along edge $(i, j)$ is exactly $X_{i j}^{*}$, as $I_{i j}=\left(U_{i}^{*}-U_{j}^{*}\right) / w_{i j}=X_{i j}^{*}>0$. (In a electrical network, the reverse current flow, i.e., the current from node $j$ to node $i$ is defined as $I_{j i}:=-I_{i j}=-X_{i j}^{*}<0$.) Hence the optimal solution to the dual problem eq.(14), $U^{*}$, is a potential function (the voltage potential in the electrical network $G$ ): $U_{i}^{*}$ is the voltage potentialfrom node $i$ to destination node $n$ (ground).

For $(i, j) \in E$, define $a_{i j}:=1 / w_{i j}$, the conductance on edge $(i, j)$, and for $(i, j) \notin E, a_{i j}=0$. From the flow conservation constraints (or directly by solving the dual optimization problem eq.(14)), we see that

$$
U_{i}^{*}=\left\{\begin{array}{ll}
\sum_{j=1}^{n} \frac{a_{i j}}{\sum_{k} a_{i k}} U_{j}^{*}+\frac{1}{\sum_{k} a_{i k}} & \text { if } i=1 \\
\sum_{j=1}^{n} \frac{a_{i j}}{\sum_{k} a_{i k}} U_{j}^{*} & \text { if } i=2, \ldots, n-1 \\
0 & \text { if } i=n
\end{array},\right.
$$

which gives the Kirchhoff's law for voltage in an electrical network. The dual problem eq.(14) gives us the Dirichlet principle [17]: the voltage potentials, $U^{*}$, taken within the electrical network $G$ minimizes the total energy dissipation. Likewise, the $L_{2}$-norm flow optimization problem also has a physical interpretation (Thompson's Principle [17]): among all flows $X \in \mathcal{F}$, the optimal (current) flow, $X^{*}$, minimizes the energy dissipation in the (electrical) network.

This connection between currents (and voltage) in electrical networks and $L_{2}$-norm network flow optimization is well known in the literature (see, e.g., [8], [13], [17], [18], [34]), where the expected round-trip commute times between two nodes in a random walk over a network, whose link weights are conductances (reciprocals of resistances), is the same as the effective resistance between the those two nodes treating the graph as an electrical network. These connections give rise to potential-based ("all-path") routing (or "stochastic routing") in communication and wireless sensor networks [17], [26]. Using the relations eq.(15) and eq.(16), it is easy to see that for any path $P$ from node 1 (source) to node $n$ (destination) in the network $G$, the (current) flow along $P$ is nonzero (i.e., $X_{i j}^{*}>0, \forall\langle i, j\rangle \in P$ ) if and only if the potential (voltage) at any node $i$ along the path from node 1 to node $n$ is strictly decreasing (i.e., $\forall\langle i, j\rangle \in P, U_{i}^{*}>U_{j}^{*}$ ). Hence the routing graph $G_{X^{*}}$ induced by the optimal flow to the $L_{2}$-norm flow minimization problem is a DAG consisting of any path from source node 1 to destination node $n$ with strictly decreasing potentials - that is what we also refer to the potential-based routing as "all-path" routing. Moreover, Ohm's law specifies how flows along the paths are split - proportional to the potential difference along an edge and inverse to the resistance of the edge, namely, $X_{i j}^{*}=\left(U_{i}^{*}-U_{j}^{*}\right) / w_{i j}$.

\section{Mixed $L_{1}$ And $L_{2}$-Norm Network Flow Op- TIMIZATION AND THE ROUTING CONTINUUM}

The results in the previous section show that the optimal flows that minimize the (weighted) $L_{1}$-norm and $L_{2}$-norm in a network yield the shortest path and (potential-based) "all-path" routing, respectively. Intuitively, if we treat $w_{i j}$ as "delay" on each link $(i, j)$, then the $L_{1}$-norm minimization produces an optimal flow routing that minimizes the total delay; whereas the $L_{2}$-norm minimization produces an optimal flow routing that minimizes the total energy dissipation (treating $w_{i j}$ as the resistance of link $(i, j))$. This gives rise to a natural question: can we generate other routing strategies between these two extremes, e.g., routing using shortest paths as well as secondshortest paths, via network flow optimization with respect to some other forms of cost metrics? In particular, can these routing strategies be derived by trading off the total delay (the $L_{1}$-norm) and the total energy (the $L_{2}$-norm)? This leads us to posing the following mixed $L_{1}$ - and $L_{2}$-norm network flow optimization problem with $\theta \geq 0$, subject to flow conservation law eqs.(7) and (8), denoted as $X \in \mathcal{F}$.

Mixed $L_{1}$ - and $L_{2}$-norm Network Flow Optimization (Primal):

$$
\min _{X \in \mathcal{F}} \sum_{i=1}^{n} \sum_{j=1}^{n} w_{i j} X_{i j}^{2}+2 \theta \sum_{i=1}^{n} \sum_{j=1}^{n} w_{i j} X_{i j}
$$

The objective function of the above mixed network flow optimization problem consists of a linear combination between the $L_{1}$ - and $L_{2}$-norm via the tradeoff parameter $\theta \geq 0$. Alternatively, the mixed objective function can be designed as a convex combination between $L_{1}$ - and $L_{2}$-norm, i.e., $(1-\lambda) \sum_{i=1}^{n} \sum_{j=1}^{n} w_{i j} X_{i j}^{2}+\lambda \sum_{i=1}^{n} \sum_{j=1}^{n} w_{i j} X_{i j}$, with $0 \leq$ $\lambda \leq 1$, which leads to exactly the same problem as eq.(18), by taking the relation $\lambda=1 /(1+2 \theta)$. In the paper, we focus on the linear combination form objective function for brevity.

Theorem 1 below presents the dual and optimal solution to this flow optimization problem, by introducing Lagrange multipliers $-2 U_{i}$.

\section{THEOREM 1. Mixed $L_{1}$ - and $L_{2}$-norm Network Flow} Optimization (Dual):

$$
\begin{aligned}
& \max _{U} U_{1}-\frac{1}{2} \sum_{i} \sum_{j: U_{i}-U_{j}>\theta w_{i j}} \frac{\left(U_{i}-U_{j}-\theta w_{i j}\right)^{2}}{w_{i j}} \\
& \text { s.t. } U_{n}=0 .
\end{aligned}
$$

Let $X^{*}(\theta)$ be the optimal solution to the primal problem eq.(18), and $U^{*}(\theta)$ the optimal solution to the dual problem eq.(19). $X^{*}(\theta)$ and $U^{*}(\theta)$ follow the following relations.

$$
X_{i j}^{*}(\theta)= \begin{cases}\frac{U_{i}^{*}(\theta)-U_{j}^{*}(\theta)}{w_{i j}}-\theta & \text { if } U_{i}^{*}(\theta)-U_{j}^{*}(\theta)>\theta w_{i j} \\ 0 & \text { if } U_{i}^{*}(\theta)-U_{j}^{*}(\theta) \leq \theta w_{i j} .\end{cases}
$$


Proof : By introducing Lagrangian multiplier $2 U_{i}(1 \leq i \leq n)$ for each equality constraint in eq.(7), and Lagrangian multiplier $2 t_{i j}(1 \leq i, j \leq n)$ for each inequality constraint in eq.(8), the Lagrangian function of the problem eq.(18) can be written as

$$
\begin{aligned}
\mathcal{L}(X, U, t) & =\sum_{i} \sum_{j}\left(X_{i j}^{2} w_{i j}-2\left(U_{i}-U_{j}-\theta w_{i j}+t_{i j}\right) X_{i j}\right) \\
& +2\left(U_{1}-U_{n}\right) .
\end{aligned}
$$

Then, we take the partial derivative of $\mathcal{L}(X, U, t)$ (eq.(22)) with respect to $X_{i j}$, and solve the equation that the partial derivative equals 0 for each $i, j=1, \ldots, n$.

$$
\begin{aligned}
\frac{\partial \mathcal{L}(X, U, t)}{\partial X_{i j}} & =2\left(X_{i j} w_{i j}-\left(U_{i}-U_{j}-\theta w_{i j}+t_{i j}\right)\right)=0 \\
X_{i j} & =\frac{U_{i}-U_{j}+t_{i j}}{w_{i j}}-\theta .
\end{aligned}
$$

Plugging the eq.(24) into eq.(22) yields the following dual problem.

$$
\begin{aligned}
& \max _{U} U_{1}-\frac{1}{2} \sum_{i} \sum_{j} \frac{\left(U_{i}-U_{j}-\theta w_{i j}+t_{i j}\right)^{2}}{w_{i j}} \\
& \text { s.t. } U_{n}=0 \text { and } \mathrm{t}_{i j} \geq 0, \text { for } i, j=1, \ldots, n,
\end{aligned}
$$

Since the primal problem is convex, the strong duality and complementary slackness hold, thus the Karush-Kuhn-Tucker (KKT) conditions [7] are sufficient and necessary to be the optimal solution to both of the primal and dual problems. The KKT conditions include the primal constraints eq.(7)-(8) and the following three conditions.

$$
\begin{aligned}
& t_{i j} \geq 0, \\
& t_{i j} X_{i j}=0, \\
& X_{i j} w_{i j}-\left(U_{i}-U_{j}-\theta w_{i j}+t_{i j}\right)=0 .
\end{aligned}
$$

From the eq.(28), $X_{i j}$ or $t_{i j}$ cannot both be zero. By setting one of them to be zero, we can solve the other. Then by checking the positivity of the solution, we get the optimal solution.

$$
\begin{aligned}
t_{i j}^{*} & = \begin{cases}0 & \text { if } U_{i}^{*}-U_{j}^{*}>\theta w_{i j}, \\
-\left(U_{i}^{*}-U_{j}^{*}-\theta w_{i j}\right) & \text { if } U_{i}^{*}-U_{j}^{*} \leq \theta w_{i j},\end{cases} \\
X_{i j}^{*} & = \begin{cases}\frac{U_{i}^{*}-U_{j}^{*}}{w_{i j}}-\theta & \text { if } U_{i}^{*}-U_{j}^{*}>\theta w_{i j}, \\
0 & \text { if } U_{i}^{*}-U_{j}^{*} \leq \theta w_{i j} .\end{cases}
\end{aligned}
$$

Since the optimal $t_{i j}^{*}$ is a function of $U_{i}^{*}$ 's, we can plug it in eqs.(25)-(26) to simply the dual problem, and eliminate the variable $t_{i j}$, which yields eq.(21).

\subsection{Optimal trade-off}

Now, we are in a position to prove that the mixed $L_{1}$ - and $L_{2}$ norm network flow optimization problem and its solution (in eq.(18) and Theorem 1) reflect the optimal trade-off between the shortest path routing and the "all path" routing, namely, for a given average delay (upper) bound, the optimal solution in Thoerem 1 leads to the minimal energy consumption, and vice versa.

Given a unit network flow from node 1 to node $n$, let $y$ be a given average delay bound as a constraint, that is, the distribution of the flow in the network yields a $L_{1}$-norm objective (the average delay) less than or equal to $y$. Then, the problem is to find the optimal flow distribution that minimizes $L_{2}$-norm objective (the energy consumption). This problem can be formulated as follows.

\section{$L_{1}$-norm constrained $L_{2}$-norm network flow optimiza-} tion problem(Primal):

$$
\begin{aligned}
& \min _{X \in \mathcal{F}} \sum_{i=1}^{n} \sum_{j=1}^{n} w_{i j} X_{i j}^{2} . \\
& \text { s.t. } \sum_{i=1}^{n} \sum_{j=1}^{n} w_{i j} X_{i j} \leq y
\end{aligned}
$$

LEMMA 2. The $L_{1}$-norm constrained $L_{2}$-norm network flow optimization problem in eq.(32) and (33) is equivalent to the mixed $L_{1}$ - and $L_{2}$-norm problem in eq.(18).

Proof Sketch: Let $2 U_{i}, 2 t_{i j}$, and $2 \theta$ be the lagrange multipliers for the flow conservation constraints eq.(8), $X_{i j} \geq 0$, and the inequality (33), respectively. Then, by the KKT condition, the dual problem is obtained as follows.

$L_{1}$-norm constrained $L_{2}$-norm network flow optimization problem(Dual):

$$
\begin{aligned}
& \max _{U, \theta} U_{1}-\frac{1}{2} \sum_{i} \sum_{j: U_{i}-U_{j}>\theta w_{i j}} \frac{\left(U_{i}-U_{j}-\theta w_{i j}\right)^{2}}{w_{i j}} \\
& \text { s.t. } U_{n}=0 \text { and } \sum_{i=1}^{n} \sum_{j=1}^{n} w_{i j} X_{i j} \leq y .
\end{aligned}
$$

The optimal solutions to the dual problem, denoted as $U^{*}(\theta)$, and $\theta^{*}$, can be obtained as following relations.

$$
\begin{gathered}
\sum_{j: U_{i}^{*}-U_{j}^{*} \geq \theta w_{i j}}\left(\frac{U_{i}^{*}-U_{i}^{*}}{w_{i j}}-\theta^{*}\right)= \begin{cases}1 & i=1 \\
0 & 1<i<n,\end{cases} \\
U_{n}^{*}=0, \text { and } \sum_{i=1}^{n} \sum_{j=1}^{n}\left(U_{i}^{*}-U_{i}^{*}-\theta^{*} w_{i j}\right)=y .
\end{gathered}
$$

From the strong duality and the complementary slackness, we have the optimal solution for the primal problem as

$$
X_{i j}^{*}(\theta)= \begin{cases}\frac{U_{i}^{*}-U_{j}^{*}}{w_{i j}}-\theta^{*} & \text { if } U_{i}^{*}-U_{j}^{*}>\theta^{*} w_{i j} \\ 0 & \text { if } U_{i}^{*}-U_{j}^{*} \leq \theta^{*} w_{i j} .\end{cases}
$$

where $\theta^{*}$ can be obtained by solving the dual problem in eq.(36) and (37), in terms of $y$. Hence, the $L_{1}$-norm constrained $L_{2}$-norm network flow optimization problem has exactly the same optimal solution of $X_{i j}^{*}$ as the mixed $L_{1}$ and $L_{2}$-norm network flow optimization problem, where the trade-off parameter $\theta^{*}$ is governed by the $L_{1}$-norm (or average delay) constraint $y$, which in turn illustrates that both problems are identical.

Similarly, given a certain average energy consumption bound, the problem of minimizing the average delay, is also equivalent to the mixed $L_{1}$ - and $L_{2}$-norm optimization problem, with the trade-off parameter $\theta^{*}$ determined by the average consumption constraint. 


\subsection{Routing Continuum}

Clearly, $\theta=0$ gives us the $L_{2}$-norm network flow optimization. In the following we will show that for sufficiently large $\theta$, the routing graph induced by the optimal solution to eq.(18) gives the same shortest path DAG as the $L_{1}$-norm flow optimization. In other words, for sufficiently large $\theta$, the optimal solution to eq.(18) yields the shortest path routing. Furthermore, for $\theta$ in between, the optimal solution to eq.(18) yields a continuum of routing graphs with the "all-path" and shortest-path DAGs as two extremes in the continuum.

Fix $\theta \geq 0$, and let $G_{X^{*}(\theta)}$ denote the routing graph (DAG) induced by the optimal flow solution $X^{*}(\theta)$ to eq.(18), i.e., for any edge $(i, j) \in E$, the $\operatorname{arc}\langle i, j\rangle$ is included in $G_{X^{*}(\theta)}$ if and only $X_{i j}^{*}(\theta)>0$. We use $P \in G_{X^{*}(\theta)}$ to denote a path $P$ from node 1 (source) to node $n$ (destination) where the flow along this path is nonzero, i.e., for any $\langle i, j\rangle \in P, X_{i j}^{*}(\theta)>0$. We have the following lemma:

LEMMA 3. Consider any path $P \in G_{X^{*}(\theta)}$, and $Q$ be any path from node 1 to node $n$. The following holds:

$$
\theta \sum_{\langle i, j\rangle \in P} w_{i j}<U_{1}^{*}(\theta) \leq(\theta+1) \sum_{\langle i, j\rangle \in Q} w_{i j} .
$$

Proof : For any $\langle i, j\rangle \in P$, since $X_{i j}^{*}(\theta)>0$, from eq.(21) we have $U_{i}^{*}(\theta)-U_{j}^{*}(\theta)=\theta w_{i j}+w_{i j} X_{i j}^{*}(\theta)$. Therefore $\sum_{\langle i, j\rangle \in P}\left(U_{i}^{*}(\theta)-U_{j}^{*}(\theta)\right)=\sum_{\langle i, j\rangle \in P}\left(\theta w_{i j}+w_{i j} X_{i j}^{*}(\theta)\right)$. Hence

$$
U_{1}^{*}(\theta)=\sum_{\langle i, j\rangle \in P}\left(\theta w_{i j}+w_{i j} X_{i j}^{*}(\theta)\right)>\theta \sum_{\langle i, j\rangle \in P} w_{i j},
$$

as $X_{i j}^{*}>0$ for any $\langle i, j\rangle \in P$. On the other hand, for any $\langle i, j\rangle \in Q$, from eq.(21) we have $U_{i}^{*}(\theta)-U_{j}^{*}(\theta) \leq \theta w_{i j}+$ $w_{i j} X_{i j}^{*}(\theta)$, where the inequality holds when $X_{i j}^{*}(\theta)=0$. Summing up along all edges $\langle i, j\rangle \in Q$, we have

$$
U_{1}^{*}(\theta) \leq \sum_{\langle i, j\rangle \in Q}\left(\theta w_{i j}+w_{i j} X_{i j}^{*}(\theta)\right) \leq(\theta+1) \sum_{\langle i, j\rangle \in Q} w_{i j},
$$

where the last inequality follows from the fact that $X_{i j}^{*} \leq$ 1. Combining this and the inequality in eq.(40) proves the lemma.

From Lemma 3, the following holds for any $\theta>0$,

$$
\sum_{\langle i, j\rangle \in P} w_{i j}<\left(1+\theta^{-1}\right) \min _{Q} \sum_{\langle i, j\rangle \in Q} w_{i j} .
$$

Using this Lemma, we establish the following theorem.

THEOREM 2 (Routing Continuum). Let $\mathcal{R}(0)$ denote the (potential-based) "all-path" routing graph in Section 2.3, namely, the routing graph induced by the optimal $L_{2}$-norm flow $X^{*}(0)$, the optimal solution to eq.(18) with $\theta=0$. Let $\mathcal{P}$ denote the collection of all paths (with nonzero flow), $P \in$ $\mathcal{R}(0)$, from source node 1 to destination $n$. Sort and group the paths based on their length, i.e., $|P|:=\sum_{\langle i, j\rangle \in P} w_{i j}$, which yields a partition (equivalent classes) of $\mathcal{P}: \mathcal{P}_{1}, \ldots, \mathcal{P}_{M}$, where $\mathcal{P}_{m}=\left\{P \in \mathcal{R}(0):|P|=L_{m}\right\}, m=1, \ldots, M$, and $L_{1}<\ldots<L_{M}$. Clearly $L_{1}$ is the length of the shortest paths.
For $\theta>0$, let $\mathcal{R}(\theta)$ denote the routing graph induced by the optimal flow $X^{*}(\theta)$, the solution to the mixed $L_{1}$ - and $L_{2}$ norm flow optimization problem. Then for $\left(L_{m}-L_{1}\right) / L_{1}<$ $\theta^{-1} \leq\left(L_{m+1}-L_{1}\right) / L_{1}, m=1, \ldots, M$ (here we define $L_{M+1}=\infty$ ), we have

$$
\mathcal{R}(\theta) \subseteq \cup_{k=1}^{m} \mathcal{P}_{k}
$$

In other words, paths in $\mathcal{R}(\theta)$ have length at most $L_{m}$.

Proof : We prove by contradiction. Given any $m, m=$ $1, \ldots, M$, and $\theta>0$ where $\left(L_{m}-L_{1}\right) / L_{1}<\theta^{-1} \leq$ $\left(L_{m+1}-L_{1}\right) / L_{1}$, suppose there exists $P \in \mathcal{R}(\theta)$ such that $|P|>L_{m}$ (thus $|P| \geq L_{m+1}$ ). From Lemma 3, the length of any path in the routing graph $\mathcal{R}(\theta)$ used to route the optimal flow $X^{*}(\theta)$ is less than $\left(1+\theta^{-1}\right) L_{1} \leq L_{m+1}$. This leads to a contradiction.

Theorem 2 states as $\theta$ increases from 0 to $\infty$, or equivalently $\theta^{-1}$ decreases to 0 , longer paths in $\mathcal{R}(0)$ are pruned, yielding a "sparser" routing graph $\mathcal{R}(\theta)$ that contains only paths of length less than $\left(1+\theta^{-1}\right) L_{1}$. In fact, there are a finite sequence of routing graphs $\mathcal{R}_{m}, 1 \leq m \leq M$, where $\mathcal{R}_{m}$ only contains paths of length at most $L_{m}$. We refer to this sequence of routing graphs as the routing continuum.

In the next section we will present an algorithm for explicitly constructing the routing continuum, and in particular, for computing the optimal flow solution, $X^{*}(\theta)$, which specifies how the optimal flow is routed among the paths in $\mathcal{R}_{m}$.

\section{Computing the Routing Continuum}

In this section we describe an efficient algorithm for computing the routing continuum and the associated optimal flow $X^{*}(\theta)$ for all $\theta$ 's, and use two simple examples to illustrate the algorithm and results obtained thereof.

We introduce an iterative process for computing the routing continuum and the optimal flow $X^{*}(\theta)$, starting with $\theta=0$, where each step involves solving a set of linear equations in $U_{i}^{*}(\theta)$ 's. Below, we provide detailed derivations of how to compute the routing continuum of a given graph, which in turn serve as a formal proof of the correctness of our proposed algorithm.

For any $\theta$, let $\mathcal{R}(\theta)=(V(\theta), E(\theta))$ denote the routing graph induced by $X^{*}(\theta)$, a subgraph of $G=(V, E)$, where $(i, j) \in \mathcal{R}(\theta)$ if and only if $X_{i j}^{*}(\theta)>0$. In the following, we will treat $\mathcal{R}(\theta)$ as an undirected graph. Hence an edge $(i, j) \in \mathcal{R}(\theta)$ if and only if either $X_{i j}^{*}(\theta)>0$ or $X_{j i}^{*}(\theta)>0$, or equivalently, $(i, j) \in \mathcal{R}(\theta)$ if and only if $\left|U_{i}^{*}(\theta)-U_{j}^{*}(\theta)\right|>$ $\theta w_{i j}$. For $i \in V(\theta)$, let $\Delta_{i}^{(+)}(\theta)$ denote the number of edges $(k, i)$ with incoming flow (i.e., $X_{k i}^{*}(\theta)>0$ ); or formally, $\Delta_{i}^{(+)}(\theta):=\sum_{k} \mathbf{1}\left\{U_{k}^{*}(\theta)-U_{i}^{*}(\theta)>\theta w_{k i}\right\}$. Likewise, let $\Delta_{i}^{(-)}(\theta)$ denote the number of edges $(i, j)$ with outgoing flow (i.e., $X_{i j}^{*}(\theta)>0$ ); thus $\Delta_{i}^{(-)}(\theta):=\sum_{j} \mathbf{1}\left\{U_{i}^{*}(\theta)-U_{j}^{*}(\theta)>\right.$ $\left.\theta w_{i j}\right\}$. Define $\Delta_{i}(\theta):=\Delta_{i}^{(-)}(\theta)-\Delta_{i}^{(+)}(\theta)$, and $d_{i}(\theta)=$ $\sum_{j:\left|U_{i}-U_{j}\right|>\theta w_{i j}} a_{i j}=\sum_{j:(i, j) \in E(\theta)} a_{i j}$, where $a_{i j}:=w_{i j}^{-1}$ if $w_{i j}>0$, and $a_{i j}:=0$ if otherwise. Then from eq.(19) (by letting the first order derivative of the objective function equal to zero), we see that the optimal $U_{i}^{*}(\theta)$ 's satisfy the following 
conditions:

$d_{i}(\theta) U_{i}^{*}(\theta)-\sum_{j:(i, j) \in E(\theta)} a_{i j} U_{j}^{*}(\theta)-\theta \Delta_{i}(\theta)= \begin{cases}1 & i=1 \\ 0 & 1<i<n,\end{cases}$

and $U_{n}^{*}(\theta)=0$.

We can rewrite eq.(43) more compactly in the matrix form:

$$
L(\theta) U^{*}(\theta)-\theta \Delta(\theta)=\mathrm{b} .
$$

Here, $L(\theta):=\left[L_{i j}(\theta)\right]$ is the $n-1$ by $n-1$ submatrix of the standard graph Laplacian [10] of $\mathcal{R}(\theta)$ (with the adjacency matrix $\left.A(\theta):=\left[a_{i j}\right], i, j \in V(\theta)\right)$, restricted to $V(\theta)-\{n\}$, namely, $L_{i i}(\theta):=d_{i}(\theta)$, and $L_{i j}(\theta):=-a_{i j}, i, j \in V(\theta)-$ $\{n\} . \Delta(\theta):=\left[\Delta_{i}(\theta)\right]$, the vector consisting of $\Delta_{i}(\theta)$, and $\mathrm{b}=[1,0, \ldots, 0]^{T}$ is the vector corresponding to the right hand side of eq.(43). Since $\mathcal{R}(\theta)$ is connected, $L(\theta)$ is non-singular and thus

$$
U^{*}(\theta)=L^{-1}(\theta)(\theta \Delta(\theta)+\mathrm{b}) .
$$

Hence given $\theta$, we can explicitly solve for $U^{*}(\theta)$ using eq.(46). However, the definitions of both $L(\theta)$ and $\Delta(\theta)$ hinge on the routing graph $\mathcal{R}(\theta)=(V(\theta), E(\theta))$, which is itself defined assuming we know $X_{i j}^{*}(\theta)$ !

This circular dependency fortunately can be broken. From Theorem 2, we know that there exist only a finite sequence of routing graphs, $\mathcal{R}\left(\theta_{m}\right), 0 \leq m \leq M$, where $0=\theta_{0}<\theta_{1}<$ $\ldots<\theta_{M}$. In other words, for $\theta_{m} \leq \theta<\theta_{m+1}, 0 \leq m \leq M$ (and define $\left.\theta_{M+1}=\infty\right), \mathcal{R}(\theta)=\mathcal{R}\left(\theta_{m}\right)$. Hence if we know $\mathcal{R}\left(\theta_{m}\right)$, we can solve $U^{*}(\theta)$ for any $\theta_{m} \leq \theta<\theta_{m+1}$ and thus $X^{*}(\theta)$. This leads to the following recursive process for computing the routing continuum and $X^{*}(\theta)$ for all $\theta \geq 0$.

\section{Phase 1: from $\theta_{0}=0$ to $\theta_{1}$ :}

When $\theta=0\left(=\theta_{0}\right), L(0)$ is $n-1$ dimensional square submatrix of the graph Laplacian on the original network $G$ (restricted to $V-\{n\}$ ). Then $U^{*}(0)=L^{-1}(0) \mathrm{b}$ is the optimal solution to the $L_{2}$-norm flow optimization, and $\mathcal{R}(0)$ is the "all-path" routing graph induced by the optimal $L_{2}$-norm flow $X^{*}(0)$.

Now consider any sufficient small $\theta>0$ (any $\theta<\theta_{1}$ would suffice) such that $\mathcal{R}(\theta)=\mathcal{R}(0)$ (thus $X_{i j}^{*}(\theta)>0$ for any $(i, j) \in \mathcal{R}(0)$. Hence $\Delta(\theta)=\Delta(0), L(\theta)=L(0)$, and $U^{*}(\theta)$ is given by

$$
U^{*}(\theta)=L^{-1}(0)(\theta \Delta(0)+\mathrm{b})=U^{*}(0)+\theta L^{-1}(0) \Delta(0) .
$$

From eq.(21) and eq.(47), if $U_{i}(\theta)-U_{j}(\theta)>\theta w_{i j}$,

$$
X_{i j}^{*}(\theta)=\frac{U_{i}^{*}(\theta)-U_{j}^{*}(\theta)}{w_{i j}}-\theta=X_{i j}^{*}(0)-\theta \alpha_{i j}(0),
$$

where $\alpha_{i j}(0)=1-\left(\beta_{i}(0)-\beta_{j}(0)\right) / w_{i j}$ is a constant, with $\beta_{i}(0)=\left[L^{-1}(0) \Delta(0)\right]_{i}$, if $i \in V-\{n\}$; and $\beta_{i}(0)=0$, if $i=n$. Eq.(47) shows that $U^{*}(\theta)$ is linear function of $\theta$, and for any edge $(i, j)$ where $X_{i j}^{*}(0)>0, X_{i j}^{*}(\theta)$ is also linear in $\theta$. Clearly, on edge $(i, j)$ with $\alpha_{i j}(0)>0$, the optimal flow $X_{i j}^{*}(\theta)$ decreases when $\theta$ increases; whereas on those with $\alpha_{i j}(0)<0$, the optimal flow $X_{i j}^{*}(\theta)$ increases $(\theta$ has no impact on those edges with $\alpha_{i j}(0)=0$ ). Hence we know precisely the (first) boundary condition, namely, the smallest positive $\theta$, when the first set of edges are to be truncated from $\mathcal{R}(0)$, namely, those where $X_{i j}^{*}(\theta)$ becomes 0 :

$$
\theta_{1}:=\min _{\langle i, j\rangle: \alpha_{i j}(0)>0}\left\{X_{i j}^{*}(0) / \alpha_{i j}(0)\right\} .
$$

Removing these edges yields the next routing graph $\mathcal{R}\left(\theta_{1}\right)$, for which $L\left(\theta_{1}\right)$ and $\Delta\left(\theta_{1}\right)$ can now be defined. Using eqs.(47) and (48), we can solve for the optimal solution, $U^{*}\left(\theta_{1}\right)$, and consequently, $X^{*}\left(\theta_{1}\right)$.

\section{Phase 2: from $\theta_{k}$ to $\theta_{k+1}$ :}

More generally, given $\mathcal{R}\left(\theta_{k}\right)$, and the corresponding optimal solutions, $U^{*}\left(\theta_{k}\right)$ and $X^{*}\left(\theta_{k}\right)$, we can solve for $U^{*}(\theta)$ and $X^{*}(\theta)$ for any $\theta_{k} \leq \theta<\theta_{k+1}$, using a similar argument. Again from eq.(46), with $L(\theta)=L\left(\theta_{k}\right)$ and $\Delta(\theta)=\Delta\left(\theta_{k}\right)$, we have

$$
\begin{aligned}
U^{*}(\theta) & =L^{-1}\left(\theta_{k}\right)\left(\theta \Delta\left(\theta_{k}\right)+\mathrm{b}\right) \\
& =U^{*}\left(\theta_{k}\right)+\left(\theta-\theta_{k}\right) L^{-1}\left(\theta_{k}\right) \Delta\left(\theta_{k}\right),
\end{aligned}
$$

and if $U_{i}(\theta)-U_{j}(\theta)>\theta w_{i j}$,

$$
X_{i j}^{*}(\theta)=\frac{U_{i}^{*}(\theta)-U_{j}^{*}(\theta)}{w_{i j}}-\theta=X_{i j}^{*}\left(\theta_{k}\right)-\left(\theta-\theta_{k}\right) \alpha_{i j}\left(\theta_{k}\right),
$$

where $\alpha_{i j}\left(\theta_{k}\right)=1-\left(\beta_{i}\left(\theta_{k}\right)-\beta_{j}\left(\theta_{k}\right)\right) / w_{i j}$ is a constant, with $\beta_{i}\left(\theta_{k}\right)=\left[L^{-1}\left(\theta_{k}\right) \Delta\left(\theta_{k}\right)\right]_{i}$, if $i \in V\left(\theta_{k}\right)-\{n\}$; and $\beta_{i}\left(\theta_{k}\right)=0$, if $i=n$. This gives us the next boundary, $\theta_{k+1}$, for the next set of links to be truncated (from $\mathcal{R}\left(\theta_{k}\right)$ ), where

$$
\theta_{k+1}=\min _{\langle i, j\rangle: \alpha_{i j}\left(\theta_{k}\right)>0}\left\{X_{i j}^{*}\left(\theta_{k}\right) / \alpha_{i j}\left(\theta_{k}\right)\right\}+\theta_{k} .
$$

Removing these edges from $\mathcal{R}\left(\theta_{k}\right)$ yields $\mathcal{R}\left(\theta_{k+1}\right)$, using which we can then solve for the optimal solutions, $U^{*}\left(\theta_{k+1}\right)$ and $X^{*}\left(\theta_{k+1}\right)$.

A pseudo-code algorithm for computing the boundary conditions $\theta_{m}$ 's, $0 \leq m \leq M$, is given in Algorithm 1, and for computing the optimal flow solution, $X^{*}(\theta)$, is given in Algorithm 2.

Complexity analysis. Since each step of the recursive process involves solving a set of linear equations [33], the worse case complexity of which is $O\left(n^{3}\right)$, and $M$ is at most $|E|$ (the number of edges), the worst-case complexity of computing the entire routing continuum is $O\left(n^{3}|E|\right)$, or $O\left(n^{5}\right)$ in the worst case.

In [25], we provide some numerical results from two synthetic networks and a real network, illustrating how the routing continuum grows as the parameter $\theta$ changes.

\section{Generalizations and Applications}

In this section, we present some extensions to the mixed $L_{1} / L_{2}$-norm network flow optimization, and briefly touch on their potential applications to traffic engineering and wireless sensor networks. In [25], we also discuss how to apply the routing continuum theory to analyze network robustness. 


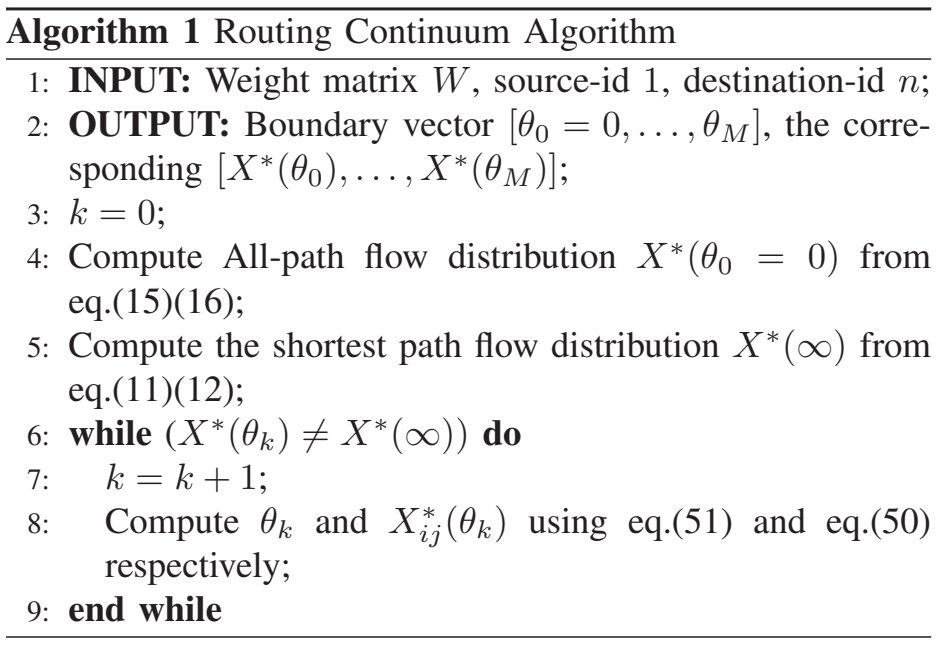

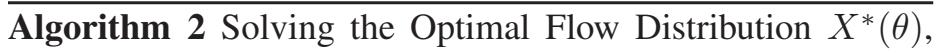
for a $\theta \geq 0$.

1: INPUT: $\left[\theta_{0}=0, \ldots, \theta_{M}\right],\left[X^{*}\left(\theta_{0}\right), \ldots, X^{*}\left(\theta_{M}\right)\right], \theta, W$, source-id 1 , destination-id $n$;

2: OUTPUT: Optimal flow distribution $X^{*}(\theta)$;

3: Search for the interval, such that $\theta \in\left[\theta_{k}, \theta_{k+1}\right)$;

4: Compute the $X^{*}(\theta)$ from eq.(50), for $\theta$.

\subsection{Multiple Flows, Link/node Capacity Constraints and Traffic Engineering}

In the previous sections, for simplicity we have assumed a single flow of unit 1 from source node 1 to destination $n$. The formulation can be easily extended to accommodate multiple flows [29], [32], [35] between different source-destination pairs and with different units, as flows are additive on (links of) the network. Consider $K$ flows, where the $k$-th flow $X^{(k)}$ of $I^{(k)}$ units is routed from source node $s_{k}$ to destination node $t_{k}, 1 \leq k \leq K$. Thus each flow $X^{(k)}$ satisfies the following conservation constraints:

$$
\sum_{j:(i, j) \in E} X_{i j}^{(k)}-\sum_{l:(l, i) \in E} X_{l i}^{(k)}= \begin{cases}I^{(k)} & \text { if } i=s_{k}(\mathrm{Src}) \\ -I^{(k)} & \text { if } i=t_{k}(\mathrm{Dst}) \\ 0 & \text { if } i \neq s_{k}, t_{k}\end{cases}
$$

We use $\mathcal{F}_{k}$ to denote the collection of flows satisfying eq.(52). Then the mixed $L_{1} / L_{2}$-norm multi-flow optimization is given in eq.(53). It is not too hard to see that this problem can be decomposed into $K$ subproblems, each of which forms a single-flow mixed $L_{1}$ and $L_{2}$-norm optimization problem, and thus can be solved using the method presented before.

$$
\min _{\substack{X^{(k)} \in \mathcal{F}_{k} \\ 1 \leq k \leq K}} \sum_{k=1}^{K} \sum_{i=1}^{n} \sum_{j=1}^{n}\left(w_{i j} X_{i j}^{(k)^{2}}+2 \theta w_{i j} X_{i j}^{(k)}\right)
$$

subject to $X_{i j}^{(k)} \geq 0,1 \leq i, j \leq n, 1 \leq k \leq K$.

In addition to having multiple flows (demands), many practical network flow problems, e.g., traffic engineering in a data network, also impose the link capacity constraints [14], [19]. Namely, given a network $G=(V, E)$, for each edge $(i, j) \in E$, let $C_{i j}\left(=C_{j i}\right)$ denote the link capacity. Then the total amount of flows on link $(i, j)$ cannot exceed $C_{i j}$.
Given any set of $K$ flows, $X^{(k)} \in \mathcal{F}_{k}, 1 \leq k \leq K$, let $\alpha$ be a variable representing the maximum link utilization in the network, i.e., $\sum_{k} X_{i j}^{(k)} \leq \alpha C_{i j}$. Similar to [35], we consider the following maximum link utilization optimization and mixed $L_{1} / L_{2}$-norm flow optimization with link capacity constraints (where $\epsilon=\theta^{-1}$ ):

Capacity Constrained Mixed Flow Optimization (Prime):

$$
\begin{aligned}
& \min _{\substack{X^{(k)} \in \mathcal{F}_{k} \\
1 \leq k \leq K}} \alpha+\sum_{k=1}^{K} \sum_{i=1}^{n} \sum_{j=1}^{n}\left(\frac{\epsilon}{2} w_{i j} X_{i j}^{(k)^{2}}+w_{i j} X_{i j}^{(k)}\right) \\
& \text { subject to } X_{i j}^{(k)} \geq 0,1 \leq k \leq K ; \text { and } \\
& \sum_{k=1}^{K} X_{i j}^{(k)} \leq C_{i j} \alpha, 1 \leq i, j \leq n .
\end{aligned}
$$

Let $U_{i}^{(k)}$ be the Lagrange multipliers for the flow conservation constraints eq.(52), and $s_{i j}$ the Lagrange multipliers for the inequality constraints $\sum_{k=1}^{K} X_{i j}^{(k)}-C_{i j} \alpha \leq 0$. Then the dual problem is given by

\section{Capacity Constrained Mixed Flow Optimization (Dual):}

$$
\begin{aligned}
& \max _{U, \mathrm{~s}} \sum_{k=1}^{K} I^{(k)} U_{1}^{(k)} \\
& -\frac{1}{2} \sum_{k=1}^{K} \sum_{i=1}^{n} \sum_{j: U_{i}^{(k)}-U_{j}^{(k)}>w_{i j}+s_{i j}} \frac{\left(U_{i}^{(k)}-U_{j}^{(k)}-\left(w_{i j}+s_{i j}\right)\right)^{2}}{\epsilon w_{i j}} \\
& \text { subject to } s_{i j} \geq 0, \sum_{i=1}^{n} \sum_{j=1}^{n} s_{i j} C_{i j}=1, \text { and } U_{n}^{(k)}=0 .
\end{aligned}
$$

Let $\alpha^{*}$ and $X_{i j}^{(k)^{*}}$ 's be the optimal solution to the primal problem and $U_{i}^{(k)^{*}}$ 's and $s_{i j}^{*}$ 's the optimal solution to the dual problem. Then by the complementary slackness, we have $X_{i j}^{(k)^{*}}>0$ if and only if $U_{i}^{(k)_{i}^{*}}-U^{(k)^{*}} \geq w_{i j}+s_{i j}^{*}$; and furthermore, if $s_{i j}^{*}>0$, then $\sum_{k=1}^{K} X^{(k)^{*}}{ }_{i j}=C_{i j} \alpha^{*}$. The latter implies that any link $(i, j) \in E$ with $s_{i j}^{*}>0$ is a "bottleneck" link where the (optimal) maximum link utilization is attained. We see that on a bottleneck link $(i, j)$, if $X^{(k)^{*}}{ }_{i j}>0$, then $X^{(k)}{ }_{i j}^{*}=\left(U^{(k)}{ }_{i}^{*}-U^{(k)_{j}^{*}}-\left(w_{i j}+s_{i j}^{*}\right)\right) /\left(\epsilon w_{i j}\right)$; whereas on a non-bottleneck link (i.e., $s_{i j}^{*}=0$ ), if $X^{(k)^{*}}{ }_{i j}>0$, then $X^{(k)^{*}}=\left(U^{(k)_{i}^{*}}-U^{(k)_{j}^{*}}-w_{i j}\right) /\left(\epsilon w_{i j}\right)$.

Comparing this with the optimal flow solutions to the mixed $L_{1} / L_{2}$-norm without the capacity constraints, an additional $s_{i j}^{*} /\left(\epsilon w_{i j}\right)$ amount is reduced from each flow $X^{(k)^{*}}{ }_{i j}$ on the bottleneck links $(i, j)$. Intuitively, it is as if the weights on the bottleneck links were replaced with $w_{i j}^{\prime}=w_{i j}+s_{i j}^{*}$ to discourage and shift away flows on the bottleneck links. In fact, suppose $s_{i j}^{*}$ 's are known a priori. We can convert the network flow optimization eq.(54) with link capacity constraints to one (without link capacity constraints) as eq.(53), where $w_{i j}$ 's in the $L_{1}$-norm term are replaced by $w_{i j}^{\prime}:=w_{i j}+s_{i j}^{*}$ 's, but not those in the $L_{2}$-norm term. This yields an example of network flow optimization with heterogenous $L_{1} / L_{2}$ costs to be discussed in the next subsection. Intuitively, this implies that the optimal flow with link capacity constraints that minimizes overall maximum link utilization is the one that discourages 
the usage of bottleneck links by increasing the $\left(L_{1}\right)$ link costs on these links and thus shifting flows away from them.

Finally, for each $\epsilon\left(=\theta^{-1}\right)$, we can use the optimal solution $\alpha^{*}(\theta)$ to eq.(54) to determine the best trade-offs between using shorter paths and longer paths, namely, the best routing graph $\mathcal{R}(\theta)$ which minimizes the overall network link utilization $\alpha(\theta)$ among any choice of $\theta \geq 0$ :

$$
\theta^{*}:=\operatorname{argmin}_{\theta \geq 0} \alpha^{*}(\theta) .
$$

In general, with the link capacity constraints, finding the optimal $\theta^{*}$ requires search in the solution space, $\left\{\theta: \alpha^{*}(\theta)\right\}$. On the other hand, assuming that $w_{i j}$ 's are fixed, we can find the optimal $\theta^{*}$ in polynomial time by first computing the entire routing continuum using Algorithms 1 and 2, and then calculating the corresponding maximum link utilization $\alpha^{*}(\theta):=\max _{(i, j)}\left\{\sum_{k} X^{(k)^{*}}{ }_{i j} / C_{i j}\right\}$ for each $\theta>0$. Thus with respect to a fixed set of link weights $w_{i j}$ 's, the routing graph $\mathcal{R}\left(\theta^{*}\right)$ yields the best trade-offs in usage of shorter and long paths: it minimizes the overall network utilization among all routing graphs.

Moreover, practical network flow problems, e.g., routing in bandwidth constrained wireless networks, may involve node capacity constraints [6], [12], [37], where for node $i \in V$, with node capacity $C_{i}$, the total amount of flows going through node $i$ cannot exceed $C_{i}$. For a set of $K$ flows, $X^{(k)} \in \mathcal{F}_{k}$, $1 \leq k \leq K, \sum_{k} \sum_{j} X_{i j}^{(k)} \leq \xi C_{i}$ holds true, where $\xi$ is the maximum node capacity utilization in the network. While considering node capacity constrained mixed $L_{1} / L_{2}$-norm flow optimization problem, similar results can be obtained as the link capacity constrained $L_{1} / L_{2}$-norm optimization problem. We omit the details here for brevity.

\subsection{Flow Optimization with Heterogeneous $L_{1} / L_{2}$ Link Weights}

We consider the following generalization where $L_{1}$-norm and $L_{2}$-norm have different sets of link weights, $w_{i j}$ 's and $r_{i j}$ 's:

Flow Optimization with Heterogeneous $L_{1} / L_{2}$ Weights (Prime):

$$
\begin{aligned}
& \min _{\substack{X^{(k)} \in \mathcal{F}_{k} \\
1 \leq k \leq K}} \sum_{k=1}^{K} \sum_{i=1}^{n} \sum_{j=1}^{n}\left(r_{i j} X_{i j}^{(k)^{2}}+2 \theta w_{i j} X_{i j}^{(k)}\right), \\
& \text { subject to } X_{i j}^{(k)} \geq 0,1 \leq k \leq K .
\end{aligned}
$$

We have already seen one instance of such generalization in the application of traffic engineering with link/node capacity constraints. Another application arises more naturally in wireless sensor networks, where deciding on the best strategies hinge on trading off different cost considerations [22], e.g., transmission latency as well as energy consumption - the latter is important, for example, to maximize the sensor network life time, where it is shown in [26] that potential-based routing using $L_{2}$-norm maximizes the network life time. Let $w_{i j}$ 's denote the per-hop transmission latency, and $r_{i j}$ 's be the transmission energy costs. Then, eq.(58) represents the mixed $L_{1} / L_{2}$-norm network flow optimization problem with heterogeneous $L_{1} / L_{2}$ link weights. The dual problem can be formulated as follows:
Flow Optimization with Heterogeneous $L_{1} / L_{2}$ Weights (Dual):

$$
\max _{U, \mathrm{~s}} \sum_{k=1}^{K} I^{(k)} U_{1}^{(k)}-\frac{1}{2} \sum_{k=1}^{K} \sum_{i=1}^{n} \sum_{j:(i, j) \in E^{(k)}(\theta)} \frac{\left(U_{i}^{(k)}-U_{j}^{(k)}-\theta w_{i j}\right)^{2}}{r_{i j}}
$$

subject to $U_{n}^{(k)}=0,1 \leq k \leq K$,

where $E^{(k)}(\theta)$ is the edge set, link $(i, j) \in E^{(k)}(\theta)$ if and only if $U_{i}^{(k)}-U_{j}^{(k)}>\theta w_{i j}$. Let $X^{(k)^{*}}$,'s and $U^{(k)^{*}}{ }_{i}$, s be the optimal solution to the primal and dual problems, respectively. By complementary slackness, we have $X^{(k)^{*}}{ }_{i j}=$ $\left(U^{(k)^{*}}-U_{i}^{(k)^{*}}-\theta w_{i j}\right) / r_{i j}>0$ if and only if $U^{(k)^{*}}{ }_{i}^{i j}-$ $U_{j}^{(k)^{*}}>\theta w_{i j}$. Using this relation, we can generalize Lemma 3 as below:

$$
\theta \sum_{\langle i, j\rangle \in P^{(k)}} w_{i j}<U^{(k)}{ }_{1}^{*}(\theta) \leq \theta \sum_{\langle i, j\rangle \in Q^{(k)}} w_{i j}+\sum_{\langle i, j\rangle \in Q^{(k)}} r_{i j},
$$

where $P^{(k)}$ is a routing path with nonzero flow $X^{(k)^{*}}$ from source $s_{k}$ to destination $t_{k}$ (i.e., $\left.P^{(k)} \in G_{X^{(k) *}}\right)$, whereas $Q^{(k)}$ is an arbitrary (simple) path in the network $G$ from source $s_{k}$ to destination $t_{k}$. For any given $\theta \geq 0$ and $1 \leq k \leq K$, using eq.(59) we can again characterize all paths in the routing graph $\mathcal{R}^{(k)}(\theta)$ - the routing graph induced by $X^{(k)^{*}}(\theta)$ : for any $P \in \mathcal{R}^{(k)}(\theta)$, its path length, $|P|<L_{\min , 1}^{(k)}+\theta^{-1} L_{\max , 2}^{(k)}$, where $L_{\min , 1}^{(k)}:=\min _{Q^{(k)}} \sum_{\langle i, j\rangle \in Q^{(k)}} w_{i j}$ is the path length of the shortest (in terms of $L_{1}$ link weights ) paths from $s_{k}$ to $t_{k}$, and $L_{\max , 2}^{(k)}:=\max _{Q^{(k)} \in \mathcal{R}^{(k)}(0)} \sum_{\langle i, j\rangle \in Q^{(k)}} r_{i j}$ is the the path length of the longest (in terms of $L_{2}$ link weights) paths in the routing graph $\mathcal{R}^{(k)}(0)$, the routing graph induced by the optimal $L_{2}$-norm flow $X^{(k)}(0)$. Therefore, we can establish a generalized routing continuum theorem analogous to Theorem 2, yielding a finite sequence of routing graphs, $\mathcal{R}^{(k)}\left(\theta_{m}^{(k)}\right)$ 's. Furthermore, the boundary conditions for $\theta_{m}^{(k)}$ 's can be precisely characterized using a similar iterative process as presented in Section 4, and Algorithms 1 and 2 can be analogously generalized to compute the entire routing continuum and $\left\{X^{(k)^{*}}(\theta), 1 \leq k \leq K\right\}$ for all $\theta>0$. We omit the details here for brevity.

\section{Related Work}

Routing in networks has been extensively studied under practical settings, with a literature too vast to cite completely. Here we will mention a few that are most relevant. For example, the authors in [28] propose an optimization model for QoS routing protocol design with multiple $L_{1}$-norm performance objectives, where the objectives are linearly combined with tunable parameter. In the context of traffic engineering in IP data networks, the authors in [15] show that given a set of traffic demands, optimizing the link weights in a network (assuming shortest-path routing) is NP-hard, and develop heuristics. The authors in [36] propose a new link-state routing protocol PEFT that goes beyond shortest paths by allowing longer paths and splitting traffic over multiple paths with an exponential penalty on longer paths. Via convex optimization, the authors show PEFT achieves optimal traffic engineering. The studies in [2], [3], [11], [30] analyze the trade-offs between shortest path 
routing and multi-path routing in both wired and wireless network settings.

Different from earlier works, which focus on routing protocol designs for specific (wired/wireless) network scenarios, our work studies routing from a more general and theoretical perspective. It is partly inspired by the finding in [35], where motivated by traffic engineering in IP networks, the authors show that shortest path routing results from the optimal flow minimizing the $L_{1}$-norm in a network. In contrast, the optimal flow minimizing the $L_{2}$-norm in a network and its connection to currents in resistive electrical networks (and random walks on a graph) are well-known (see [17] and references thereof); it leads to the potential-based, "all-path" (or stochastic) routing that has been applied in wireless sensor networks, e.g., to maximize network life time [26], or to minimize state maintenance [9]. Our work generalizes these earlier results to show that using the mixed $L_{1} / L_{2}$-norm flow optimization, we can construct the entire routing continuum from the shortestpath to all-path, with routing graphs consisting of paths of increasing path lengths.

\section{Conclusion}

In this paper, we have formulated the network routing problem as flow optimization problem in a network with mixed $L_{1} / L_{2}-$ norms. Using this formulation, we established a surprising result: the routing graphs induced by the optimal flow solutions span the entire routing continuum from the shortest-path to allpath routing. Using the duality theory, we also developed an efficient iterative process for computing the entire routing continuum and optimal flow solutions $X^{*}(\theta)$ for any $\theta \geq 0$. The basic theory is further extended to account for multiple flows (traffic demands), link capacity constraints and heterogeneous $L_{1} / L_{2}$ link weights, with applications to traffic engineering and wireless sensor networks, and network robustness analysis.

As part of future work, we plan to investigate the routing continuum theory on directed graphs (with both uni- and bidirectional links) or signed graphs (with both positive and negative links), by applying the spectral graph theory developed for directed graphs [5], [21]-[23] and signed graphs [20].

\section{References}

[1] G. R. Ash. Dynamic Routing in Telecommunications Networks. McGraw-Hill Professional, 1st edition, 1997.

[2] C. L. Barrett, S. J. Eidenbenz, L. Kroc, M. V. Marathe, and J. P. Smith. Probabilistic multi-path vs. deterministic single-path protocols for dynamic ad-hoc network scenarios. In SAC, 2005.

[3] A. Basu, A. Lin, and S. Ramanathan. Routing using potentials: a dynamic traffic-aware routing algorithm. In SIGCOMM, New York, NY, USA, 2003.

[4] S. Biswas and R. Morris. Exor: opportunistic multi-hop routing for wireless networks. In SIGCOMM, 2005.

[5] D. Boley, G. Ranjan, and Z.-L. Zhang. Commute times for a directed graph using an asymmetric Laplacian. Linear Algebra and Appl., 435:224-242, 2011.

[6] T. Bonald, M. Jonckheere, and A. Proutiére. Insensitive load balancing. In SIGMETRICS, 2004

[7] S. Boyd and L. Vandenberghe. Convex optimization. Cambridge university press, 2004.

[8] A. K. Chandra, P. Raghavan, W. L. Ruzzo, R. Smolensky, and P. Tiwari. The electrical resistance of a graph captures its commute and cover times. In STOC, 1989.

[9] C.-K. Chau and P. Basu. Exact analysis of latency of stateless opportunistic forwarding. In INFOCOM, 2009.
[10] F. R. K. Chung and S. T. Yau. Discrete green's functions. Journal of Combinatorial Theory, Series A, pages 191-214, 2000.

[11] I. Cidon, R. Rom, and Y. Shavitt. Analysis of multi-path routing. IEEE/ACM Transactions on Networking., 7:885-896, December 1999.

[12] A. V. Donati, R. Montemanni, L. M. Gambardella, and A. E. Rizzoli. Integration of a robust shortest path algorithm with a time dependent vehicle routing model and applications. In Computational Intelligence for Measurement Systems and Applications, 2003. CIMSA '03. 2003 IEEE International Symposium on, 2003.

[13] P. Doyle and J. Snell. Random Walks and Electric Networks. The Math. Assoc. of Am., 1984. http: // front.math.ucdavis.edu/ math.PR/0001057.

[14] W. Fisher, M. Suchara, and J. Rexford. Greening backbone networks: reducing energy consumption by shutting off cables in bundled links. In Proceedings of the first ACM SIGCOMM workshop on Green networking, Green Networking '10, 2010.

[15] B. Fortz and M. Thorup. Increasing internet capacity using local search. Computational Optimization and Applications, 29(1):13-48, 2004.

[16] D. Ganesan, R. Govindan, S. Shenker, and D. Estrin. Highly-resilient, energy-efficient multipath routing in wireless sensor networks. SIGMOBILE CCR, 5(4):11-25, October 2001.

[17] F. P. Kelly. Network routing. Philosophical Transactions of the Royal Society, A(337):343-367, 1991.

[18] D. Klein and M. Randic. Resistance distance. Journal Mathematical Chemistry, 12:81-95, 1993.

[19] L. Li, M. Thottan, B. Yao, and S. Paul. Distributed network monitoring with bounded link utilization in ip networks. In INFOCOM, 2003.

[20] Y. Li, W. Chen, Y. Wang, and Z.-L. Zhang. Influence diffusion dynamics and influence maximization in social networks with friend and foe relationships. In WSDM, 2013.

[21] Y. Li, Z. Zhang, and J. Bao. Mutual or unrequited love: Identifying stable clusters in social networks with uni-and bi-directional links. WAW, 2012.

[22] Y. Li and Z.-L. Zhang. Random walks on digraphs: A theoretical framework for estimating transmission costs in wireless routing. In INFOCOM, 2010.

[23] Y. Li and Z.-L. Zhang. Random walks on digraphs, the generalized digraph laplacian and the degree of asymmetry. In WAW, 2010.

[24] Y. Li, Z.-L. Zhang, and D. Boley. The routing continuum from shortestpath to all-path: A unifying theory. In ICDCS, 2011.

[25] Y. Li, Z.-L. Zhang, and D. Boley. From shortest-path to all-path: The routing continuum theory and its applications (supplementary materials). IEEE Transactions on Parallel Distributed Systems, 2013.

[26] H. Liu, Z.-L. Zhang, J. Srivastava, and V. Firoiu. Pwave: A multi-source multi-sink anycast routing framework for wireless sensor networks. In NETWORKING, 2007.

[27] D. G. Luenberger. Linear and nonlinear programming (Second edition). Addison-Wesley, 1984.

[28] P. V. Mieghem and L. Vandenberghe. Trade-off curves for qos routing. In Infocom, 2006.

[29] M. Neely, E. Modiano, and C. Rohrs. Dynamic power allocation and routing for time varying wireless networks. In INFOCOM, 2003.

[30] P. Pham. Performance analysis of reactive shortest single-path and multipath routing mechanism with load balance. In INFOCOM, 2003.

[31] L. Popa, C. Raiciu, I. Stoica, and D. Rosenblum. Reducing congestion effects in wireless networks by multipath routing. In ICNP, 2006.

[32] P. Purkayastha and J. S. Baras. An optimal distributed routing algorithm using dual decomposition techniques. Communications in Information and Systems, 8(3):277-302, 2008.

[33] W. J. Stewart. Numerical methods for computing stationary distributions of finite irreducible markov chains. Computational Probability, pages 81-111, 1999.

[34] P. Tetali. Random walks and effective resistance of networks. Journal of Theoretical Probability, pages 101-109, 1991.

[35] Y. Wang, Z. Wang, and L. Zhang. Internet traffic engineering without full mesh overlaying. In INFOCOM, 2001.

[36] D. Xu, M. Chiang, and J. Rexford. Link-state routing with hop-by-hop forwarding can achieve optimal traffic engineering. In INFOCOM, pages 466-474, 2008.

[37] G. Zussman and A. Segall. Energy efficient routing in ad hoc disaster recovery networks. In INFOCOM, 2003. 


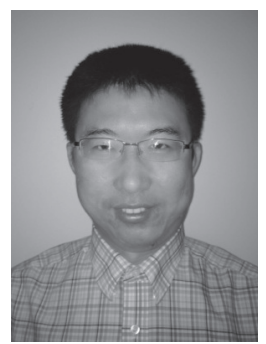

Yanhua Li (S'09-M'13) received the B.S. and M.S. degrees in electrical engineering from Sichuan University, Chengdu, China, in 2003 and 2006, and two Ph.D. degrees in electrical engineering from Beijing University of Posts and Telecommunications, Beijing in China in 2009 and in computer science from University of Minnesota at Twin Cities in 2013, respectively. He is now a researcher in HUAWEI Noah's Ark lab in China.

His research interests are wireless networking and social network analysis in many contexts, including communication protocols design and high performance networking scheduling strategies, social graph analysis and measurements.

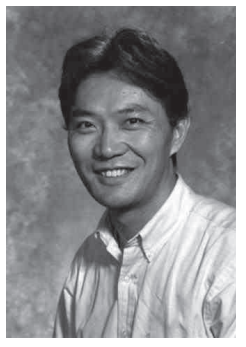

Zhi-Li Zhang (M'97-SM'11-F'12) received the B.S. degree in computer science from Nanjing University, Jiangsu, China, in 1986, and the M.S. and Ph.D. degrees in computer science from the University of Massachusetts Amherst, Amherst, in 1992 and 1997, respectively.

In 1997, he joined the Computer Science and Engineering faculty at the University of Minnesota, Minneapolis, MN, where he is currently a Professor. From 1987 to 1990, he conducted research with the Computer Science Department, Aarhus University, Aarhus, Denmark, under a fellowship from the Chinese National Committee for Education. He has held visiting positions with Sprint Advanced Technology Labs, Burlingame, CA; IBM T. J. Watson Research Center, Yorktown Heights, NY; Fujitsu Labs of America, Sunnyvale, CA; Microsoft Research China, Beijing, China; and INRIA, Sophia-Antipolis, France.

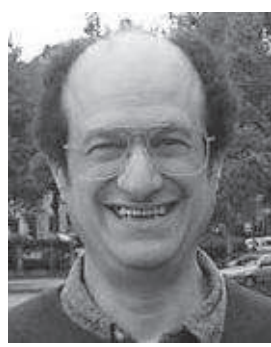

Daniel Boley received his Ph.D. degree in Computer Science from Stanford University in 1981. Since then, he has been on the faculty of the Department of Computer Science and Engineering at the University of Minnesota, where he is now a full professor. Dr. Boley is known for his past work on numerical linear algebra methods for control problems, parallel algorithms, iterative methods for matrix eigenproblems, inverse problems in linear algebra, as well as his more recent work on computational methods in statistical machine learning, data mining, and bioinformatics. His current interests include the analysis of networks and graphs such as those arising from metabolic biochemical networks and networks of wireless devices. He has been an associate editor for the SIAM Journal of Matrix Analysis and has chaired several technical symposia at major conferences. 


\title{
From Shortest-path to All-path: The Routing Continuum Theory and its applications (Supplementary Materials)
}

\author{
Yanhua Li, Member, IEEE, Zhi-Li Zhang, Fellow, IEEE, and Daniel Boley, Member, IEEE
}

\begin{abstract}
This documents provides numerical illustration results obtained by applying the routing continuum theory in two synthetic networks and a real network topology. Moreover, besides the generalizations of the mixed $L_{1}$ - and $L_{2}$-norm network flow optimization problem discussed in the main file, in this document, we discuss one more application in analyzing network robustness, by introducing the generalized shortest path and random walk betweenness centrality measures.
\end{abstract}

Index Terms-Routing continuum, network flow, betweenness centrality.

\section{Numerical Illustration of Routing Con- TINUUM THEORY}

We use two synthetic networks and a real network to show how the routing continuum grows as the parameter $\theta$ changes. Fig. 1 shows an example topology, with three disjoint paths between the source 1 and the destination 5 , and each link is with 1 -unit weight. As the parameter $\theta \geq 0$ increases, the longer paths $P 3=\{1 \rightarrow 3 \rightarrow 4 \rightarrow 5\}$ and $P 2=$ $\{1 \rightarrow 2 \rightarrow 5\}$ are truncated gradually, and the shortest path $P 1=\{1 \rightarrow 5\}$ is obtained when $\theta$ increases to 1 . Fig. 2 shows the routing continuum, i.e. the optimal flow distributions at each $\theta$. We see that within the interval $\theta \in[0,0.4]$, the flows on the longer paths $P 2$ and $P 3$ get linearly redistributed to the shortest path $P 1$, and the longest path $P 3$ gets truncated when $\theta=0.4$. Then the flow of the second longest path $P 2$ keeps decreasing as $\theta$ increases, until the second boundary condition $\theta=1$ holds, where $P 2$ is truncated. During the routing evolution process, the network flows are always redistributed from longer paths to the shorter path, while increasing $\theta$. When $\theta>1$, namely, the largest boundary condition, the routing solution is stabilized to the shortest path, i.e. $P 1$.

Fig. 3 shows another example with five connected nodes in the topology. Weights $w_{i j}$ 's are marked on the links. The flow initiates at source 1 and is removed from destination 5 . Fig. 4 Fig. 8 show the optimal flow distributions (marked on individual links) under five boundary conditions, $\left[\theta_{0}=0, \theta_{1}=\right.$ 0.0914, $\left.\theta_{2}=0.2850, \theta_{3}=0.5700, \theta_{4}=2\right]$, In Fig. $4\left(\theta_{0}=0\right)$, every link is active and follows the potential based "all-path" routing. Then, as $\theta$ increases to $\theta_{1}=0.0914$ (in Fig.5), link $(1,4)$ is truncated, and within the interval $\theta \in\left[0, \theta_{1}\right]$, only the flow on path $\{1 \rightarrow 4 \rightarrow 5\}$ decreases, and gets redistributed to other paths, because this path with total length 11 is the

- This work was supported in part by the NSF grants CNS-1017092 and IIS-0916750, the DTRA grant HDTRA1-09-1-0050. An earlier version of this work appeared in the Proceedings of IEEE ICDCS [22], June 2011. Yanhua Li is with HUAWEI Noah's Ark Lab, China. Zhi-Li Zhang and Daniel Boley are with the Department of Computer Science and Engineering, University of Minnesota, Twin Cities, Minneapolis, MN, 55455.

E-mail: \{yanhua,zhzhang,boley\}@cs.umn.edu longest path in $\mathcal{P}(0)$, i.e. the "all-path" routing graph. Then, when $\theta$ increases to $\theta_{2}=0.2850$, the flows on links $(2,3)$ and $(3,5)$ are truncated, because these two links are on the second longest path $\{1 \rightarrow 2 \rightarrow 3 \rightarrow 5\}$, with path length 5. Similarly, when $\theta$ keeps increasing to $\theta_{3}$ and $\theta_{4}$, the rest two longer paths $\{1 \rightarrow 2 \rightarrow 5\}$ and $\{1 \rightarrow 2 \rightarrow 4 \rightarrow 5\}$ get removed, respectively, and only the shortest path $\{1 \rightarrow 5\}$ is left at last.

Now, we apply the routing continuum theory to Internet 2 Abilene Network [1]. The Abilene network was a highperformance backbone network established by the Internet2 community in the late 1990s. The Abilene Network was retired and became the "Internet2 Network" in 2007. Fig. 15 shows its 11 regional network aggregation points and backbone connections across them (primarily OC192 or OC48 backbone). We consider the transmission cost between two end points roughly proportional to their actual geographic distance, because the velocity of light in an optical fiber becomes $60-70 \%$ compared to it in vacuum [13], [26], [33]. Hence, in the numerical analysis, we simply use the geographical distance as the link weight for the transmission cost as marked in Fig. 9. We choose the flow demand from Sunnyvale to New York. As we increase $\theta$ from 0 , we observe a sequence of five boundary $\theta$ 's, i.e., $\left[\theta_{0}=0, \theta_{1}=0.1082, \theta_{2}=0.2498, \theta_{3}=\right.$ $\left.0.4943, \theta_{4}=3.2108\right]$, in which order links $(4 \rightarrow 6),(5 \rightarrow 1)$, $(10 \rightarrow 9 \rightarrow 3)$ and $(10 \rightarrow 7 \rightarrow 4 \rightarrow 1 \rightarrow 11 \rightarrow 8)$ get truncated in sequence, and the optimal flow distribution evolves from the "all-path" routing to "shortest-path" routing. When $\theta_{0}=0$, all paths are present in delivering the contents, whereas only the shortest path $\{10 \rightarrow 3 \rightarrow 6 \rightarrow 5 \rightarrow 2 \rightarrow 8\}$ is active for $\theta \geq 3.2108$.

\section{Network robustness analysis Via gener- ALIZED CENTRALITY MEASURE}

The optimal flow distribution $X^{*}(\theta)$ to the mixed $L_{1}-$ and $L_{2}$-norm network flow optimization problem indicates exactly the loads on each link (resp. node) for certain flow demands. When considering flow demands from all source 


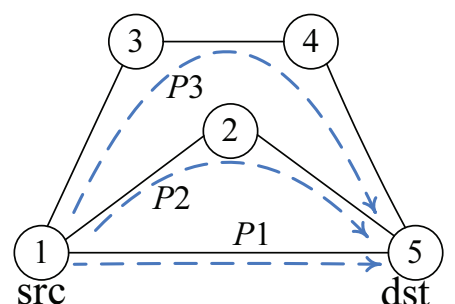

Fig. 1. Example 1 with uniform weight $w_{i j}=1$

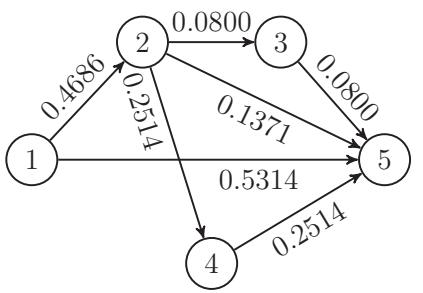

Fig. 5. Flow distribution with $\theta_{1}=0.0914$

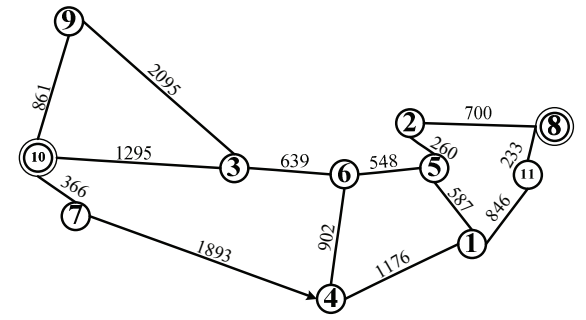

Fig. 9. Weights on the Abilene network

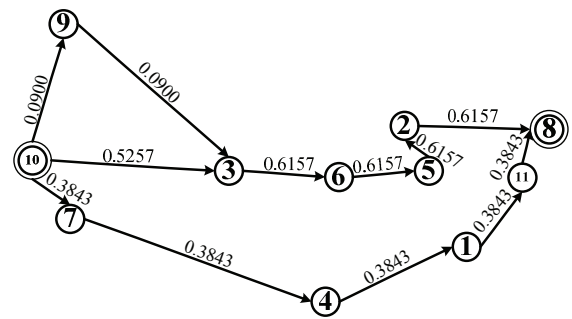

Fig. 12. Flow distribution $\theta_{1}=0.2498$

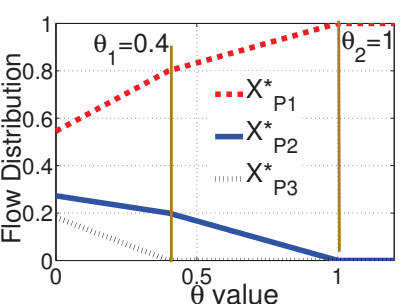

Fig. 2. Flow distribution evolution of graph Fig. 1

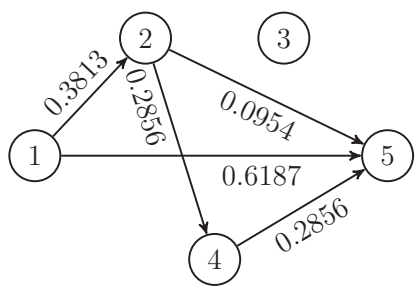

Fig. 6. Flow distribution with $\theta_{2}=0.2850$

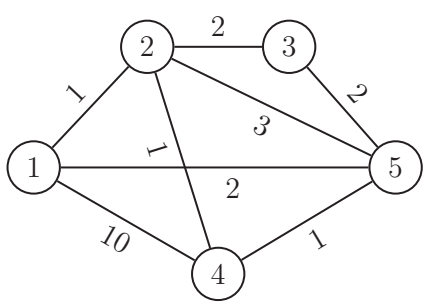

Fig. 3. Example 2: Weight Graph

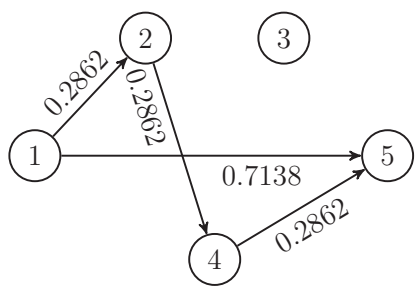

Fig. 7. Flow distribution with $\theta_{3}=0.5700$

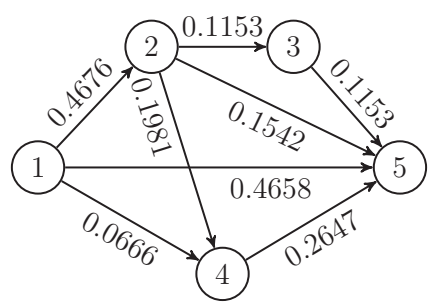

Fig. 4. Flow distribution, $\theta_{0}=0$

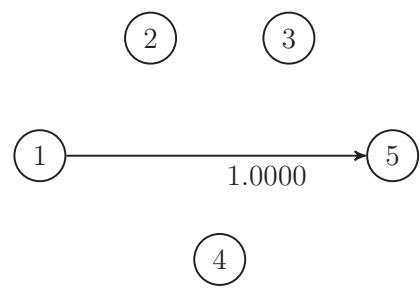

Fig. 8. Flow distribution with $\theta_{4}=2$

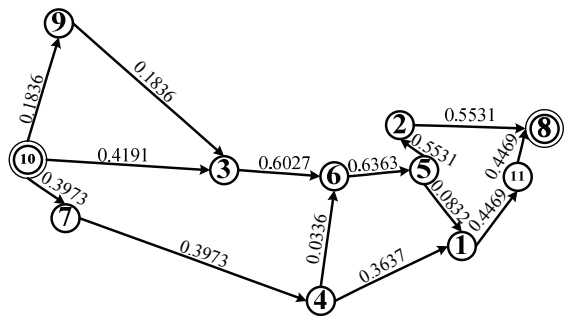

Fig. 10. Flow distribution $\theta_{0}=0$ (9)

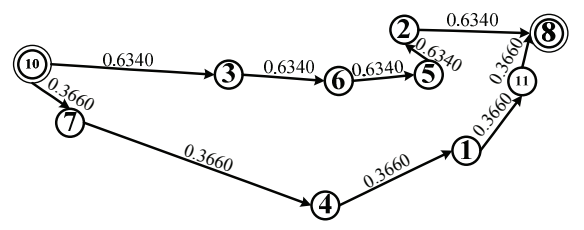

Fig. 13. Flow distribution $\theta_{3}=0.4943$

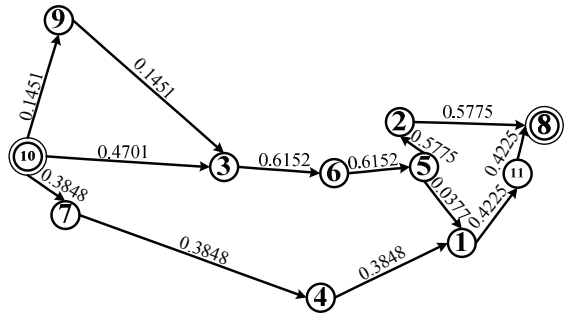

Fig. 11. Flow distribution $\theta_{1}=0.1082$ (9)

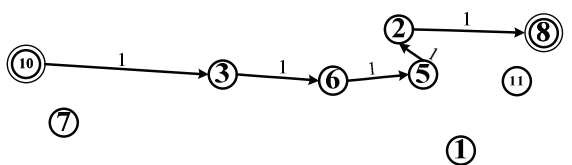

(4)

Fig. 14. Flow distribution $\theta_{4}=3.2108$ destination pairs, the average network flow on each link (resp. node) infers the "importance" of the link (resp. node), namely, the influence of the link (resp. node) in case of failure or being attacked, which in turn reveals the robustness structure of networks, i.e., which area of the network is more vulnerable to attacks. The robustness centrality measure of links and nodes in the network has been extensively studied, and has been applied to design topology control algorithm and routing protocol in wireless sensor networks and delay tolerant networks [14], [19]. Below, we show how our routing continuum theory can be used to generalize various robustness centrality measures of links/nodes in networks, where the ranking of links/nodes in terms of their betweenness infer the network robustness structure, i.e., those areas with high betweenness links/nodes expose more risks to attacks or failures, as when removing these links/nodes, more flows have to be rerouted or failed.

\subsection{Centrality measures for mixed network flow}

Centrality measures were first developed in social network analysis [9], [25], for example, how influential a user is in a social network, with applications in robust community detection [18], [23], mobility prediction [5], and etc. There are four widely used centrality measures [25], that capture the relative importance of a vertex or an edge within a network from various aspects: degree ${ }^{1}$, eigenvector centrality $^{2}$, betweenness [10], [16], and closeness [28]. Betweenness and closeness centrality measures are directly interpretable in

1. The node degree centrality is simply defined as the number of links associated with a node, which reflects locally (i.e., within one hop,) how well the node is connected to other nodes.

2. Eigenvector centrality takes the leading Eigenvector, i.e., the Eigenvector corresponding to the largest Eigenvalue, of the adjacent matrix $A$ as relative scores to all nodes in the network, which follows the concept that connections to nodes with higher scores contribute more to the score of the node than connections to nodes with lower scores. PageRank [27] and Katz centrality [20] can be viewed as two variations of the Eigenvector centrality measure. 


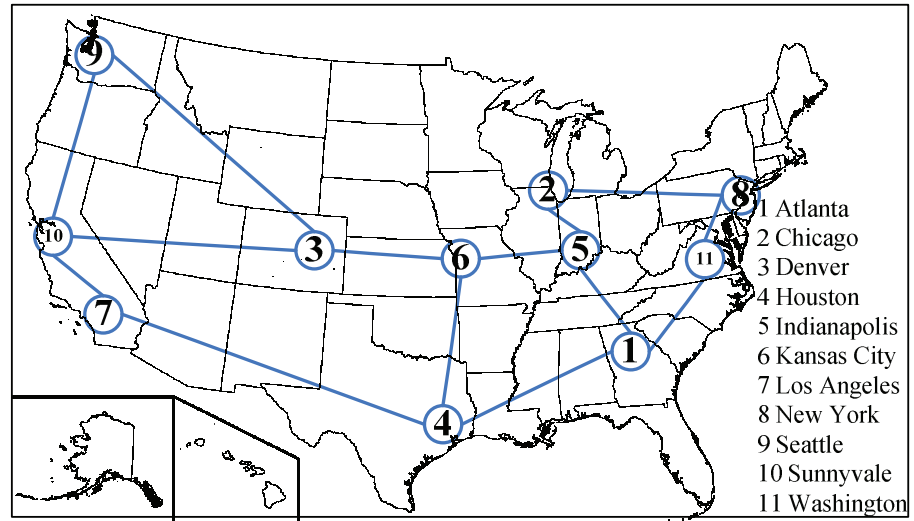

Fig. 15. Abilene network topology

terms of the shortest path and all-path routing, thus can be generalized using our routing continuum theory to account for mixed network flows. In the following, we will introduce the mixed-flow betweenness for nodes or edges, a natural generalization of the existing betweenness centrality measures. The mixed-flow betweenness measures indicate the importance of nodes or edges in terms of the degree to which a node or an edge is participating in the communication between node pairs in the network, which has implications in network resource relocations and detecting robust subgraphs that are resilient to attacks and failures. Note that closeness centrality can similarly be generalized, and we omit these results here for brevity.

\subsection{Node Betweenness centrality}

Node betweenness has been studied in the past as a measure of the centrality and influence of nodes in networks.

Shortest-path betweenness. A simple example of such a betweenness measure initially proposed by Freeman [8], [16], [17] is shortest-path betweenness. Given a node $i$, its shortest-path betweenness is defined as the number of shortest (geodesic) paths between pairs of all other nodes that run through $i$. To be precise, given a graph $G=(V, E)$, node $i$ 's betweenness centrality [16], [17] $C_{i}^{S}$ is defined ss $^{3}$

$$
C_{i}^{S}=\frac{2 \sum_{s<t \in V} g_{i}^{(s t)}}{n(n-1)}
$$

where $g_{i}^{(s t)}$ is the number of shortest paths from node $s$ to node $t$ that pass through $i$. Since the graph is undirected, $g_{i}^{(s t)}=$ $g_{i}^{(t s)}$ always holds, thus computing $g_{i}^{(s t)}$ for only half of all node pairs (i.e., for $s<t$ ) is sufficient. If there is more than one shortest path between a node pair, each path is given equal weight such that the total weight of all of the paths is unity. Since when $\theta$ is large enough, the optimal flow distribution denoted by $X^{*}(\infty)$ represents the shortest path solution, the

3. Here, the normalizing constant is $n(n-1)$, where $i$ may also be a start or end node of a source destination pair. Some definitions only count for those node pairs without $i$ as a start or end node, where the normalizing constant becomes $(n-1)(n-2)$ instead. shortest path betweenness centrality $C_{i}^{S}$ can be written as

$$
C_{i}^{S}=\frac{2 \sum_{s<t \in V} \sum_{k \in V} X^{(s t)}{ }_{k i}^{*}(\infty)}{n(n-1)} .
$$

Current-flow betweenness ${ }^{4}$. Considering that the circuit created by placing a resister on each edge of the network and unit current source and destination at a particular node pair. The resulting current flow in the network will follow Kirchhoff's and Ohm's laws, going from source to destination along a multitude of paths. Hence, The current-flow betweenness [24] for a node $i$ is defined as the absolute value of the currents summed over all node pairs that run through $i$. The optimal optimal flow distribution $X^{(s t)^{*}}(0)$ of the $L_{2}$ norm flow optimization problem represents exactly the current flow for source destination pair $(s, t)$ with $\theta=0$. The current-flow betweenness $C_{i}^{C}$ of node $i$ can be written in terms of $X^{(s t)^{*}}(0)$ as

$$
C_{i}^{C}=\frac{2 \sum_{s<t \in V} \sum_{k \in V} X_{k i}^{(s t)^{*}}(0)}{n(n-1)} .
$$

Mixed-flow betweenness. Shortest-path betweenness and current-flow betweenness present two extremes. One uses only shortest paths, and the other favors all-path to deliver network flow. Our routing continuum theory naturally leads to a generalized mix-flow betweenness, $C_{i}(\theta)$, which captures how much mixed flow $X^{*}(\theta)$ runs through a node given a flow combination parameter $\theta$.

$$
C_{i}(\theta)=\frac{2 \sum_{s<t \in V} \sum_{k \in V} X_{k i}^{(s t)}{ }_{k i}^{*}(\theta)}{n(n-1)},
$$

with $\theta \geq 0$. Note that the shortest-path betweenness (eq.(2)) and the current-flow betweenness (eq.(3)) are two special cases of mixed-flow betweenness, as $C_{i}^{S}=C_{i}(\infty)$ and $C_{i}^{C}=C_{i}(0)$, respectively. Given a specific $\theta \geq 0, C_{i}(\theta)$ captures the importance of node $i$, in terms of the average optimal flow going through node $i$ over all source destination pairs.

\subsection{Edge betweenness centrality}

Analogically, the betweenness centrality can be defined for edges, capturing how much network flow going through a particular edge, summed over all node pairs in the network.

The shortest-path betweenness of an edge $(i, j)$ is the total number of shortest paths running along $(i, j)$, which was first introduced by Anthonisse in [6], and Newman formally defined it in [23]. It can be written in terms of the optimal shortest path flow distribution denoted by $X^{*}(\infty)$ as

$$
C_{i j}^{S}=\frac{2 \sum_{s<t \in V} X^{(s t)}{ }_{i j}^{*}(\infty)}{n(n-1)}
$$

Similarly, the current-flow betweenness of an edge $(i, j)$ is the current flow running along $(i, j)$ [10], [23], which can be

4. Current-flow betweenness is proven to be equivalent to random walk (RW) betweenness [24]. For a node $i$, we calculate the expected number of times that a random walk between a particular node pair will pass through $i$, and RW betweenness is the summation over all node pairs. 
computed using the following eq.(6) in terms of the optimal $L_{2}$ network flow distribution denoted by $X^{*}(0)$ as

$$
C_{i j}^{C}=\frac{2 \sum_{s<t \in V} X^{(s t)}{ }_{i j}^{*}(0)}{n(n-1)}
$$

The mixed-flow betweenness of an edge $(i, j)$ is then a natural generalization of eq.(5) and eq.(6) for $\theta \geq 0$.

$$
C_{i j}(\theta)=\frac{2 \sum_{s<t \in V} X^{(s t)}{ }_{i j}^{*}(\theta)}{n(n-1)}
$$

As discussed earlier, the trade-off parameter $\theta \geq 0$ governs how much shortest path flow vs current flow is considered in the mixed flow optimization problem. The mixed-flow betweenness centrality measure for a link/node captures how crucial the link/node is in carrying the network flow for all possible node pairs. In communication networks, the link/node betweenness measures in fact indicate how much (mixed) network flow has to go through a particular link/node for all source-destination pairs. An attack or failure to the links/nodes with high betweenness leads to more influential impacts to the network traffic. Hence the ranking of the links/nodes in terms of their betweenness infer the network robustness structure, namely, areas with high betweenness links/nodes are more vulnerable to attacks or failures, since more flows have to be rerouted or failed if these links/nodes fail.

TABLE 1

Edge ranking in mixed-flow betweenness $(\theta \geq 0)$.

\begin{tabular}{|c||c|c|c|}
\hline $\begin{array}{c}\text { Edge } \\
\text { ranking }\end{array}$ & $\begin{array}{c}\text { when } \theta \in \\
{[0,0.002)}\end{array}$ & $\begin{array}{c}\text { when } \theta \in \\
{[0.002,0.06)}\end{array}$ & $\begin{array}{c}\text { when } \\
\theta \geq 0.06\end{array}$ \\
\hline$\# 1$ & $\mathbf{( 1 , 2 )}$ & $\mathbf{( 1 , 2 )}$ & $\mathbf{( 2 , 4 )}$ \\
\hline$\# 2$ & $\mathbf{( 4 , 5 )}$ & $\mathbf{( 2 , 4 )}$ & $\mathbf{( 1 , 2 )}$ \\
\hline$\# 3$ & $\mathbf{( 2 , 4 )}$ & $\mathbf{( 4 , 5 )}$ & $\mathbf{( 4 , 5 )}$ \\
\hline$\# 4$ & $(2,3)$ & $(2,3)$ & $(2,3)$ \\
\hline$\# 5$ & $(3,5)$ & $(3,5)$ & $(3,5)$ \\
\hline$\# 6$ & $(1,5)$ & $(1,5)$ & $(1,5)$ \\
\hline$\# 7$ & $(2,5)$ & $(2,5)$ & $(2,5)$ \\
\hline$\# 8$ & $(1,4)$ & $(1,4)$ & $(1,4)$ \\
\hline
\end{tabular}

\subsection{Numerical examples}

Next, we use the topology in Fig. 3 and a real network topology, i.e., Internet2 Abilene Network [1], as examples, to show how the ranking of node/link in terms of betweenness changes over $\theta$.

When computing the betweenness centrality measures for the five node topology in Fig. 3, we observe that as increasing $\theta \geq 0$, the ranking of links in terms of their mixed-flow betweenness keeps relatively robust, namely, there are only three different link ranking orders (See Tab 1). The highest betweenness links are $(1,2),(4,5)$ and $(2,4)$, which all have the smallest link weights. The link $(2,4)$ steps up to the highest ranking, when $\theta \geq 0.06$. The node betweenness ranking is more stable, which is unchanged over $\theta$ 's for topology in Fig. 3 with nodes ranked as $\{2,5,4,1,3\}$ in a decreasing order. Nodes with more links and lower link weights are ranked higher, since they are more likely to serve as hubs to carry more network flows.
Now we investigate how the link and node betweenness ranking vary over $\theta$ in Abilene network. As we increase $\theta \geq 0$, there are twelve boundary $\theta$ 's, governing the different ranking of link betweenness in Abilene network as shown in Table 2. We observe that when $\theta$ changes, namely, the network flow evolves from "all-path" flow to "shortest-path" flow, the ranks of links with the highest betweeness keep high ranking over $\theta$, i.e., links at rank \# 1 to \# 5 are unchanged. On the other hand, the betweenness centrality link $(3,10)$ increases from the rank \#11 to \#6 gradually (as highlighted in Table 2), which happens because the high link weight of $(3,10)$ suppress the "all-path (current)" flow going through it, but it resides on more shortest paths among node pairs, thus generates higher shortest-path flow when $\theta$ is large. Moreover, the ranks of links such as $(1,11),(2,8)$, and $(8,11)$ decrease as $\theta$ increases. The ranks of some other links, including $(1,5)$ and $(4,7)$, keep stable at rank \#7-\#9. When looking at the node betweenness, the ranking is more stable than links, which is unchanged for all $\theta$ 's as shown in Table 3. The nodes placed in central US, such as Kansas City and Indianapolis posses highest node betweenness centrality, namely, being the busiest nodes in carrying network flow.

We also computed the ranking of link/node betweenness in other real networks, such as Roofnet [3] (with 38 nodes), CERNET [4] (with 36 nodes), GEANT [2] (with 23 nodes), where similar results are obtained and we omit them here for brevity. From all these results, the node betweenness centrality ranking is overall more stable than link betweenness centrality ranking, through the entire routing continuum, i.e., all $\theta \geq 0$.

\section{Discussion}

In a broader context, the mixed $L_{1} / L_{2}$ optimization formulation has been widely used, e.g., in the classical LASSO problems [30], namely, the least square optimization problems with a $L_{1}$-norm penalty term, and more recently, in compressive sensing [11], [31]. It is therefore well-known that the $L_{1}$-norm penalty forces the least-square solution, $X^{*}$, to meet certain sparsity constraints, i.e., $\left\|X^{*}\right\|_{1} \leq \epsilon$. Compared with LASSO and compressive sensing settings, our setting has a set of additional flow conservation constraints - these are what makes the problem unique and leads to solutions that have interesting interpretations and consequences, where the solutions to the more general LASSO and compressive sensing settings may not have, apart from the sparsity of the solutions.

Indeed, our routing continuum theory and the mixed $L_{1}$ and $L_{2}$-norm flow optimiaztion can be interpreted in terms of the "sparsity" of the solutions also: the optimal flow solution $X^{*}(\theta)$ to the mixed $L_{1} / L_{2}$-norm flow optimization leads to a sparser routing graph, where the path length of routes used for routing the optimal flow from a source to a destination can not be $\left(1+\theta^{-1}\right)$ longer than the shortest paths. More surprising and interesting is that we can generate the entire routing continuum from the mixed $L_{1} / L_{2}$-norm flow optimization. The flow conservation constraints in fact play a key role here: it leads to the duality of the optimal flows, $X^{*}(\theta)$, a function defined on the edges of a network, and the optimal (generalized) potential functions, $U^{*}(\theta)$, a function defined on the nodes of a network. This allows us to solve $U^{*}(\theta)$ through 
TABLE 2

Edge ranking in mixed-flow betweenness in Abilene network $(\theta \geq 0)$.

\begin{tabular}{|c|c|c|c|c|c|c|c|c|c|c|c|c|}
\hline$\theta$ & 0 & 0.0535 & 0.0617 & 0.0647 & 0.0839 & 0.0918 & 0.0964 & 0.1079 & 0.1335 & 0.2650 & 0.2874 & 0.3176 \\
\hline \# 1 & $(5,6)$ & $(5,6)$ & $(5,6)$ & $(5,6)$ & $(5,6)$ & $(5,6)$ & $(5,6)$ & $(5,6)$ & $(5,6)$ & $(5,6)$ & $(5,6)$ & $(5,6)$ \\
\hline$\# 2$ & $(3,6)$ & $(3,6)$ & $(3,6)$ & $(3,6)$ & $(3,6)$ & $(3,6)$ & $(3,6)$ & $(3,6)$ & $(3,6)$ & $(3,6)$ & $(3,6)$ & $(3,6)$ \\
\hline \#3 & $(2,5)$ & $(2,5)$ & $(2,5)$ & $(2,5)$ & $(2,5)$ & $(2,5)$ & $(2,5)$ & $(2,5)$ & $(2,5)$ & $(2,5)$ & $(2,5)$ & $(2,5)$ \\
\hline$\# 4$ & $(7,10)$ & $(7,10)$ & $(7,10)$ & $(7,10)$ & $(7,10)$ & $(7,10)$ & $(7,10)$ & $(7,10)$ & $(7,10)$ & $(7,10)$ & $(7,10)$ & $(7,10)$ \\
\hline \# 5 & $(1,4)$ & $(1,4)$ & $(1,4)$ & $(1,4)$ & $(1,4)$ & $(1,4)$ & $(1,4)$ & $(1,4)$ & $(1,4)$ & $(1,4)$ & $(1,4)$ & $(1,4)$ \\
\hline \# 6 & $(2,8)$ & $(2,8)$ & $(2,8)$ & $(2,8)$ & $(2,8)$ & $(2,8)$ & $(2,8)$ & $(2,8)$ & $(2,8)$ & $(2,8)$ & $(3,10)$ & $(3,10)$ \\
\hline \# 7 & $(8,11)$ & $(8,11)$ & $(8,11)$ & $(8,11)$ & $(4,7)$ & $(4,7)$ & $(1,5)$ & $(1,5)$ & $(1,5)$ & $(3,10)$ & $(2,8)$ & $(1,5)$ \\
\hline$\# 8$ & $(4,7)$ & $(4,7)$ & $(4,7)$ & $(4,7)$ & $(8,11)$ & $(1,5)$ & $(4,7)$ & $(4,7)$ & $(3,10)$ & $(1,5)$ & $(1,5)$ & $(2,8)$ \\
\hline \# 9 & $(1,11)$ & $(1,5)$ & $(1,5)$ & $(1,5)$ & $(1,5)$ & $(8,11)$ & $(8,11)$ & $(3,10)$ & $(4,7)$ & $(4,7)$ & $(4,7)$ & $(4,7)$ \\
\hline$\# 10$ & $(1,5)$ & $(1,11)$ & $(1,11)$ & $(3,10)$ & $(3,10)$ & $(3,10)$ & $(3,10)$ & $(8,11)$ & $(8,11)$ & $(8,11)$ & $(8,11)$ & $(8,11)$ \\
\hline \# 11 & $(3,10)$ & $(3,10)$ & $(3,10)$ & $(1,11)$ & $(1,11)$ & $(1,11)$ & $(1,11)$ & $(1,11)$ & $(1,11)$ & $(1,11)$ & $(1,11)$ & $(1,11)$ \\
\hline
\end{tabular}

TABLE 3

Node betweenness ranking in the Abilene network for all $\theta$ 's.

\begin{tabular}{|c|c|c|c|c|c|c|c|c|c|c|}
\hline Rank \# 1 & $\# 2$ & $\# 3$ & $\# 4$ & $\# 5$ & $\# 6$ & $\# 7$ & $\# 8$ & $\# 9$ & $\# 10$ & $\# 11$ \\
\hline Kansas City & Indianapolis & Denver & Atlanta & Sunnyvale & Houston & Chicago & Los Angeles & New York & Washington & Seattle \\
\hline
\end{tabular}

a set of linear equations, and yields an efficient process to compute the entire routing continuum and the optimal flow $X^{*}(\theta)$ for any $\theta \geq 0$. Last but not the least, we remark that although we only focus on network routing in this paper, we believe that our results can be applied to many other applications where the problems can be cast in terms of flows in a network.

Another line of works that is related to our study is parametrized dissimilarity measure (or distance) between nodes. Yen et al. [32] develop a family of link-based dissimilarity measures, namely, the randomized shortest-path (RSP) dissimilarity, which generalizes both the weighted shortest path distance and the resistance distance. It is interpreted as the path probability distribution that minimizes the expected energy for transiting from a source node to a destination node, constrained by a fixed relative entropy (Kullback-Leibler divergence) with respect to the reference probability. Chebotarev [12] introduces a similar parametric family of node distance to [32] by matrix forest theorem and the transition inequality, which possess a unique graph-geodetic property: $d(i, j)+d(j, k)=d(i, k)$ if and only if every path from $i$ to $k$ passes through $j$. Different from our work, the constraints exploited in these works are no longer flow conservation law, thus the solutions obtained have different interpretations of the underlying "flow".

Various algorithms have been proposed to identify the potential based "all-paths" and the shortest paths for a given node pair in the literature. The former [21] requires solving a linear equation system [29] with $O\left(n^{3}\right)$, where $n$ is the total number of nodes in the graph. On the other hand, Dijkstras algorithm [15] and Bellman-Ford algorithm [7] were introduced to compute shortest paths between a node pair in a time complexity of $O\left(n^{2}\right)$ and $O(n m)$ (roughly $O\left(n^{3}\right)$ when the graph is dense), respectively, with $m$ as the total number of edges in the graph. These algorithms aim to extract a routing graph (i.e., the flow distribution) for a particular $\theta$, where our routing continuum algorithm computes the complete set of routing graphs between shortest path routing and "all-path" routing by scanning all possible $\theta$ 's, thus taking at the worst case $O\left(n^{3} m\right)$. However, in many applications with relatively stable link status, the set of routing graphs do not need to be computed frequently, e.g., in wireless sensor networks with static link transmission delay and energy consumption, and in intradomain administrative network with small chance of link failure. It is interesting to develop more efficient algorithm that can better deal with dynamic link weights, where we leave this as part of our future work.

\section{REFERENCES}

[1] Abilene Project by Internet2 community. http://www.internet2.edu/abilene/.

[2] (GN3) GEANT Project. http://www.geant.net/pages/home.aspx.

[3] Mit roofnet project. http://pdos.csail.mit.edu/roofnet/.

[4] The China Education and Research Network (CERNET). http://www.edu.cn/cernet_1377/index.shtml.

[5] Y. Altshuler, R. Puzis, Y. Elovici, S. Bekhor, and A. S. Pentland. Augmented betweenness centrality for mobility prediction in transportation networks. In NEMO' 11: Proceedings of the international workship on finding patterns of human behaviors in network and mobility data, 2011.

[6] J. M. Anthonisse. The rush in a directed graph. Technical Report BN 9/71, Stichting Mathematisch Centrum, Amsterdam, 1971.

[7] R. Bellman. On a routing problem. Quarterly of Applied Mathematics, 16:87-90, 1958.

[8] S. Borgatti and M. Everett. A graph-theoretic perspective on centrality. Social networks, 28(4):466-484, 2006.

[9] U. Brandes and T. Erlebach. Network analysis: methodological foundations, volume 3418. Springer, 2005.

[10] U. Brandes and D. Fleischer. Centrality measures based on current flow. STACS 2005, pages 533-544, 2005.

[11] E. J. Candes and M. B. Wakin. An Introduction To Compressive Sampling. IEEE Signal Processing Magazine, 25(2):21-30, March 2008.

[12] P. Chebotarev. A class of graph-geodetic distances generalizing the shortest-path and the resistance distances. Discrete Applied Mathematics, 159(5):295-302, 2011.

[13] S. Cheshire. Latency survey results (for "it's the latency, stupid"), 1996.

[14] A. Cuzzocrea, A. Papadimitriou, D. Katsaros, and Y. Manolopoulos. Edge betweenness centrality: A novel algorithm for qos-based topology control over wireless sensor networks. Journal of Network and Computer Applications, 35(4):1210-1217, 2012.

[15] E. W. Dijkstra. A note on two problems in connexion with graphs. Numerische mathematik, 1:269-271, 1959.

[16] L. C. Freeman. A set of measures of centrality based on betweenness. Sociometry, 40(1):35-41, March 1977.

[17] L. C. Freeman. Centrality in social networks conceptual clarification. Social Networks, 1(3):215-239, 1979.

[18] M. Girvan and M. Newman. Community structure in social and biological networks. Proceedings of the National Academy of Sciences, 99(12):7821-7826, 2002.

[19] G. Grossi and F. Pedersini. Hub-betweenness analysis in delay tolerant networks inferred by real traces. In Modeling and Optimization in Mobile, Ad Hoc and Wireless Networks (WiOpt), 2010 Proceedings of the 8th International Symposium on, pages 318-323. IEEE, 2010. 
[20] L. Katz. A new index derived from sociometric data analysis. Psychometrika, 18:39-43, 1953.

[21] F. P. Kelly. Network routing. Philosophical Transactions of the Royal Society, A(337):343-367, 1991.

[22] Y. Li, Z.-L. Zhang, and D. Boley. The routing continuum from shortestpath to all-path: A unifying theory. In ICDCS '11: Proceedings of the 31st International Conference on Distributed Computing Systems, pages 847-856. IEEE, 2011.

[23] M. Newman and M. Girvan. Finding and evaluating community structure in networks. Physical review E, 69(2):026113, 2004.

[24] M. E. J. Newman. A measure of betweenness centrality based on random walks. Social Networks, 27(1):39-54, January 2005.

[25] M. E. J. Newman. Networks: An Introduction. Oxford University Press, 1st edition, 2010.

[26] K. Okamoto. Fundamentals of optical waveguides. Academic press, 2010.

[27] L. Page, S. Brin, R. Motwani, and T. Winograd. The pagerank citation ranking: Bringing order to the web. Technical report, Stanford University, 1998.

[28] G. Sabidussi. The centrality index of a graph. Psychometrika, 31:581603, 1966.

[29] W. J. Stewart. Numerical methods for computing stationary distributions of finite irreducible markov chains. Computational Probability, pages 81-111, 1999.

[30] R. Tibshirani. Regression shrinkage and selection via the lasso. Journal of the Royal Statistical Society (Series B), 58:267-288, 1996.

[31] Y. Tsaig and D. L. Donoho. Compressed sensing. IEEE Transactions on Information Theory, 52:1289-1306, 2006.

[32] L. Yen, M. Saerens, A. Mantrach, and M. Shimbo. A family of dissimilarity measures between nodes generalizing both the shortestpath and the commute-time distances. In Proceeding of the 14th ACM SIGKDD international conference on Knowledge discovery and data mining, pages 785-793. ACM, 2008.

[33] K. Yoshida, Y. Kikuchi, M. Yamamoto, Y. Fujii, K. Nagami, I. Nakagawa, and H. Esaki. Inferring pop-level isp topology through end-to-end delay measurement. Passive and Active Network Measurement, pages 35-44, 2009. 